REVIEW ARTICLE OPEN

\title{
Pathways to Parkinson's disease: a spotlight on 14-3-3 proteins
}

\author{
E. Giusto $\mathbb{D}^{1}$, T. A. Yacoubian ${ }^{2}$, E. Greggio $\mathbb{I}^{3}$ and L. Civiero $\mathbb{I}^{1,3 凶}$
}

14-3-3s represent a family of highly conserved $30 \mathrm{kDa}$ acidic proteins. 14-3-3s recognize and bind specific phospho-sequences on client partners and operate as molecular hubs to regulate their activity, localization, folding, degradation, and protein-protein interactions. 14-3-3s are also associated with the pathogenesis of several diseases, among which Parkinson's disease (PD). 14-3-3s are found within Lewy bodies (LBs) in PD patients, and their neuroprotective effects have been demonstrated in several animal models of PD. Notably, 14-3-3s interact with some of the major proteins known to be involved in the pathogenesis of PD. Here we first provide a detailed overview of the molecular composition and structural features of 14-3-3s, laying significant emphasis on their peculiar target-binding mechanisms. We then briefly describe the implication of 14-3-3s in the central nervous system and focus on their interaction with LRRK2, a-Synuclein, and Parkin, three of the major players in PD onset and progression. We finally discuss how different types of small molecules may interfere with 14-3-3s interactome, thus representing a valid strategy in the future of drug discovery.

npj Parkinson's Disease (2021)7:85; https://doi.org/10.1038/s41531-021-00230-6

\section{INTRODUCTION}

14-3-3s define a ubiquitous, highly conserved family of proteins whose name reflects the elution fraction of an ion-exchange chromatography performed by Moore and Perez in 1967, in which 14-3-3s were discovered to be major components of bovine brain lysates. Since then, 14-3-3s have been identified in all eukaryotes studied so far, spanning from unicellular organisms to mammals, although differences in their isoform-specific expression and function have emerged along the evolutionary process ${ }^{1}$. Most of the known organisms express multiple isoforms of 14-3-3s (indicated with Greek letters), each encoded by a distinct gene ${ }^{2}$. Despite early observations suggesting functional overlap, later reports established that different isoforms have acquired a certain degree of specificity (reviewed in ref. ${ }^{3}$ ). The biological relevance of $14-3-3$ s has remained underrated for over 20 years, until they emerged as crucial components in the biosynthesis of neurotransmitters in the brain ${ }^{4}$. Almost at the same time, multiple lines of evidence showed that 14-3-3s could orchestrate different biological activities within the cell, including cell cycle progres$\operatorname{sion}^{5}$, cell transformation and mitogenic signal regulation ${ }^{6,7}$, intracellular signaling ${ }^{8,9}$, and exocytosis ${ }^{10}$. However, it was not until 1996 that crystallographic studies began to reveal the hallmark structure of $14-3-3 \mathrm{~s}$ and, with this, some powerful insights into their polyhedric nature ${ }^{11}$. In particular, it was discovered that 14-3-3s were present mainly as homo- or heterodimers and that they were capable of binding numerous target proteins containing specific phospho-serine/phosphothreonine motifs ${ }^{11}$, although many exceptions also exist ${ }^{12}$. The ability of $14-3-3$ s to target such an exceptional range of "client" proteins represents the essence of their multitasking functionality and explains their critical involvement into multiple cellular activities.

Parkinson's disease (PD) offers a unique platform in this context. Indeed, 14-3-3s stand at the crossroad of several pathways involved in the pathogenesis of the disease. For example, 14-3-3 $\beta$ and $14-3-3 \gamma$ were found to be at the center of some transcriptionally deregulated pathways in PD patients ${ }^{13}$, while isoforms $-\gamma$ and $-\eta$ show the strongest affinities for leucine-rich repeats kinase 2 (LRRK2), a key protein in the onset of familial and sporadic PD (see below) ${ }^{14}$. Likewise, $14-3-3 \eta$ has been shown to regulate the ubiquitin ligase activity of Parkin and is able to reduce the formation of toxic a-synuclein aggregates in vitro, while isoforms $-\theta,-\varepsilon$, and $-\gamma$ exhibit a major neuroprotective effect in experimental models of $\mathrm{PD}^{15-17}$. Therefore, a better understanding of 14-3-3s functional network and a selective modulation of their interactions with crucial partners may represent a new strategy to disentangle the molecular roots of PD.

\section{Origin and evolution}

Multiple numbers and combinations of 14-3-3 isoforms are present in distinct classes of organisms, ranging from budding (S.pombe) and fission (S.cerevisiae) yeasts, which both express two isoforms ${ }^{18}$, to Arabidopsis, which expresses up to 15 different isoforms ${ }^{6}$. The mammalian genome host seven major transcripts $(\beta, \gamma, \varepsilon, \zeta, \eta, \theta / \tau, \sigma)$ encoded by distinct genes, while those originally described as $a-$ and $\delta$ - isoforms have finally been recognized as the phosphorylated forms of $\beta$ - and $\zeta-14-3-3 \mathrm{~s}$, respectively ${ }^{19,20}$. Studies based on sequence alignments suggest the presence of a common ancestor from which different isoforms would have diverged along with the evolution of major eukaryotic lineages. Within the animal group, the $-\varepsilon$ isoform holds the most ancestral sequence, and shares some key features with yeasts and plants, thus diverging from the remaining non- $\varepsilon$ isoforms ${ }^{21}$. Interestingly, some isoforms may share higher sequence identity (up to 96-100\%) with paralogs belonging to different species within the same group (e.g., mammalian) rather than with other isoforms in the same species (about 50\%) ${ }^{1}$.

The biological relevance of such a variety is controversial. Early studies describing an efficient replacement between 14-3-3 isoforms coming from different species seemed to support the hypothesis of functional redundancy, as expected also by the high degree of sequence homology. Indeed, the expression of plant or human-derived 14-3-3s effectively rescued the lethal phenotype caused by the double disruption of the yeast genes $B M H I$ and

IRCCS San Camillo Hospital, Venice, Italy. ${ }^{2}$ Center for Neurodegeneration and Experimental Therapeutics, Department of Neurology, University of Alabama at Birmingham, Birmingham, AL, USA. ${ }^{3}$ Department of Biology, University of Padova, Padova, Italy. ${ }^{\bowtie}$ email: laura.civiero@unipd.it 


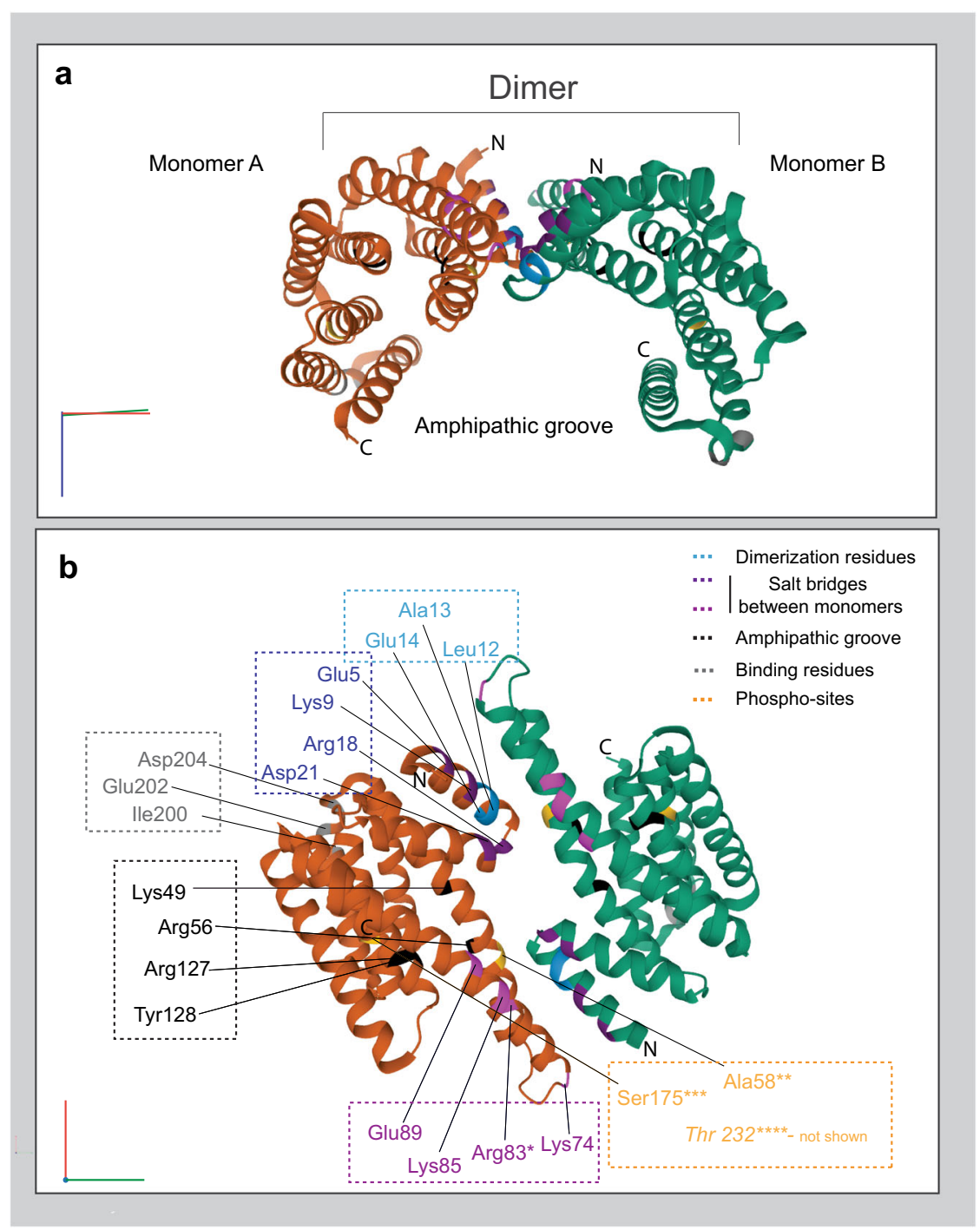

Fig. 1 Structure of 14-3-3. Orthogonal views of human $14-3-3 \theta / \tau$, modified from $6 B D 1^{226}$. a Schematic representation of the main structure of human $14-3-3 \theta / \tau$ showing the spatial allocation of the amphipathic groove. $\mathbf{b}$ Some of the most significant residues involved in structure stability and functionality. Some isoform-specific mutations are depicted: Arg83* is replaced by Glu83 in the 14-3-3 $\sigma$ isoform and involved in Lys9-Glu83 binding. Ala58** is present only in isoforms $-\theta / \tau$ and $-\sigma$; all the other isoforms present a Ser58 which is a very important phosphorylation site (see main text). Ser175*** indicated as Ser184/186 in the main text. C: C-terminal, N: N-terminal.

$B M H 2$ (coding for $14-3-3$ s in Saccharomyces cerevisiae) ${ }^{22}$. Yet, the existence of isoform-specific motifs as well as a preferential tissue or cellular tropism and a differential subcellular recruitment, suggest that a substantial degree of functional specialization does exist $^{3}$. More recently, the combination of high-throughput analyses with the availability of databases hosting considerable amounts of protein-protein interaction (PPI) data, has pointed to the concept of "adaptive subfunctionalization". According to this, mild mutations may damp the deleterious effects of dosageimbalance caused by duplication events, thus reconciling genetic redundancy and functional specialization ${ }^{23,24}$.

\section{Structural characteristics of 14-3-3s}

In homeostatic conditions, 14-3-3s exist mainly as dimers (Fig. 1a), a configuration that is believed to favor their stability (e.g., by reducing susceptibility to phosphorylation) and activity ${ }^{25}$. However, dimers can be dissociated and converted into monomers upon the phosphorylation of specific residues (see below), and certain 14-3-3 isoforms may naturally exist as monomers and dimers $^{26,27}$. Also, a defective splicing variant of $14-3-3 \varepsilon$ which lacks the N-terminal region-usually involved in dimer formation-has been recently found ${ }^{28}$.

All 14-3-3 isoforms show significant structural homologies, which in part reflect the overall degree of sequence identity. Each monomer is composed of 9 a-helices arranged in antiparallel order and connected by short loops ${ }^{29}$. In dimers, helices from both monomers interact in a symmetrical manner, in which helix a1 (amino acids 2-16) of one monomer connects with helices a3 (amino acids 38-66) and a4 (amino acids 73-103) of the opposite monomer and vice versa ${ }^{30}$. While some isoforms prefer assembling into heterodimers, others mainly form homodimers. This difference depends on the presence of 3 main salt bridges involving Arg18-Glu89, Glu5-Lys74, and Asp21-Lys85 (Fig. 1b). As an example, the $-\varepsilon$ isoform only contains one of these salt bridges. Therefore, its association with a different isoform (which inevitably contains more salt bridges) would provide higher stability to the resulting dimer than the one deriving from the association with another $-\varepsilon$ monomer. On the other end, the $\sigma$-isoform, where the Glu5-Lys74 salt bridge is replaced by the Lys9-Glu83 interaction, is more liable to form homodimers ${ }^{31}$. This aptitude of different monomers to interact further extends the repertoire of target 
proteins that can be engaged by 14-3-3 dimers. The conformational organization of dimers results in a cup-shaped pocket approximately $35 \AA$ broad, $35 \AA$ wide, and $20 \AA$ deep, meant to accommodate the target protein $(s)^{32}$. Structure analyses of $14-3-3 \mathrm{~s}$ bound to peptides have revealed that the side chains of some specific residues, namely Lys49, Arg56, Arg127, and Tyr128, confer an amphipathic nature to the groove, crucial to the binding of target proteins (Fig. 1a, b) 1,32,33. These residues outline the most inner part of the pocket and are strictly conserved in mammals and yeasts ${ }^{29}$. They form numerous hydrophobic and polar interactions, as well as salt bridges between either identical or different isoforms, thus conferring a rigid structure to the dimer, essential for its function ${ }^{34}$. Of note, these conserved residues give each isoform the same potential to bind a peptide, while variable residues that line the external surface of the groove provide the specificity of each 14-3-3:target interaction ${ }^{32}$. Despite the high degree of sequence homology at the level of the groove (over $70 \%$ between organisms $)^{35}, \mathrm{~N}$ - and $\mathrm{C}$-terminals are quite divergent between isoforms, with the main exception being represented by ${ }^{12} \mathrm{LAE}^{14}$ residues, which are conserved in all the species and crucial for the dimerization process (Fig. 1b) ${ }^{26,36}$.

Intriguingly, because of some peculiar variations in the amino acidic sequence surrounding the binding groove, its total lack of introns, its main expression in epithelial cells, and its preference at forming homodimers, the $\sigma$ isoform represents the most peculiar member within the 14-3-3 family ${ }^{37}$.

\section{Definition of consensus binding motifs}

The high level of sequence conservation of the amphipathic groove suggests that target proteins may also need specific anchoring residues to be recognized and accepted at the dimer interface. While some recurrent binding motifs have been determined, the repertoire of target proteins able to interact with $14-3-3 s$ is much more extended than initially thought. Early observations suggested that the phosphorylation of a Ser residue might have been pivotal. In support of this, Michaud et al. showed that the phosphorylation of Ser259 in the CR2 domain of Raf-1 was strictly required for its interaction with 143-3s, which could have been prevented by phosphatase treatment or by the mutated S259A-Raf ${ }^{38}$. Similarly, endogenous phosphorylation of tryptophan hydroxylase by a $\mathrm{Ca}^{2+} / \mathrm{calmo}^{-}$ dulin or CAMP-dependent kinase is essential for its binding to 14-3-3n in vitro ${ }^{39,40}$. By using a panel of degenerated peptides derived by c-Raf, Muslin and collaborators identified RSX(pS/pT) $\mathrm{XP}$ as a first putative binding motif recognized by 14-3-3s (mode I). Notably, all the isoforms are able to recognize this motif and bind with high affinity ${ }^{11}$. Similar results were obtained also by Yaffe et al., who, in addition, proposed RX(F/Y)XpSXP as a novel binding motif (mode II) ${ }^{32}$. More recently a third motif (mode III) has been identified. This consensus sequence, $\mathrm{pS} / \mathrm{TX}_{1-2}-\mathrm{COOH}$, lies at the C-terminal of target proteins, and still requires phosphorylation to bind 14-3-3s. Only a few targets containing this motif have been studied so far, and further investigations are needed to better define the most common surrounding residues. Finally, some proteins known to interact with 14-3-3s fall outside these conventional binding rules and do not strictly depend on phosphorylation events ${ }^{12}$.

The presence of two binding sites (one from each monomer) in a 14-3-3 dimer suggests that 14-3-3s may simultaneously bind different targets and function as scaffold proteins. However, it is also true that one single target protein may contain more than one binding site such that one target protein can interact with both 14-3-3 monomers. In many cases, the binding affinity of these additional sites may be too low to ensure a long-lasting interaction, as binding affinity depends on the extent to which the binding motif matches the optimal consensus sequence. Within a target protein, there is likely a dominant binding site that acts as a "gatekeeper" and favors the concomitant interaction of secondary (weaker) binding sites. In fact, an association between 14-3-3s and proteins containing tandem phospho-serine motifs can lead to a 30 -fold increase in their binding affinity ${ }^{32,41}$. In this context, Stevers and coworkers propose a thermodynamic model to describe how a delicate enthalpy/entropy balance is crucial in determining the overall binding affinity of a target protein to 14-33s. In particular, the authors analyze different, multivalent bindings for cystic fibrosis transmembrane conductance regulator (CFTR) and for the PD multi-domain kinase LRRK2, which present nine and six binding sites for 14-3-3s, respectively. Importantly, they noticed that LRRK2 peptides carrying different combinations of the 14-3-3s binding sites present different $K_{d}$ values, with the highest ones shown by pS910 and pS935. Therefore modifications of these sites may be of interest for therapies aimed at blocking or reinforcing 14-3-3:LRRK2 binding (see below) ${ }^{42}$.

\section{4-3-3s biological functions}

Because of their rigid structure, 14-3-3s are thought to work as "molecular anvils", showing little or no change in their overall conformation upon target binding. This interaction would instead force target proteins to undergo changes in their structure, thus altering some of their functional properties, localization, or PPIs ${ }^{41}$. One of the first studies supporting this theory showed that 14-3-3s stabilize the structure of phosphorylated Serotonin Acetyltransferase (AANAT), increasing its catalytic activity and substrate affinity (Fig. 2a, top box) ${ }^{43}$. 14-33s can also influence the functionality of target proteins by masking or exposing their nuclear localization motifs. This is particularly important for those proteins whose activity is controlled by their subcellular localization, such as those involved in cell cycle regulation. p27 is a cyclin-dependent kinase inhibitor which can be phosphorylated at multiple sites, including Thr157, a residue contained in its nuclear localization signal (NLS). Once bound to phosphorylated Thr157, isoforms $14-3-3 \beta,-\varepsilon$ and $-\gamma$ mask this NLS and prevent the interaction between p27 and importin- $\alpha$, with the consequent cytoplasmic withholding of p27 (Fig. 2a, second box) ${ }^{44,45}$. Alternatively, upon binding to 14-3-3s, target proteins may fail to interact with their downstream partners, as in the case of the regulator of $\mathrm{G}$ protein signaling $3\left(\mathrm{RGS}_{3}\right)^{46}$. Similarly, BAD needs to dissociate from 14$3-3 \mathrm{~s}$ to interact with phosphatase PP2A and initiate the apoptotic program (Fig. 2a, middle box) ${ }^{47}$ and ref. ${ }^{2}$.

14-3-3s can also act as scaffolds. Thanks to their ability to simultaneously bind different target proteins through their bivalent docking site, $14-3-3$ s can work as hubs to promote functional interaction between partners. As an example, 14-3-3 isoforms $-\beta$ and $-\zeta$ can form heterotrimeric complexes with Raf- 1 and protein kinase $C$ (PKC)- $\zeta$. Once the complex is formed, PKC- $\zeta$ can phosphorylate both Raf-1, which becomes active, and 14-3-3s, finally leading to the dissociation of the ternary complex (Fig. 2a, fourth box). Brief, temporary interactions like this may provide a mechanism by which cells activate dynamic signaling cascades in response to external stimuli ${ }^{48,49}$.

Another recognized, but less studied function of $14-3-3 \mathrm{~s}$ is the one related to their chaperone-like properties. While $14-3-3$ s do not meet all of the conventional criteria commonly attributed to "professional chaperones", they do participate in the regulation of protein folding and aggregation ${ }^{50-53}$. Although considered an auxiliary, "moonlighting" activity of $14-3-3 s^{54}$, it gains particular relevance for those pathologies characterized by the formation and accumulation of misfolded proteins, such as Alzheimer's disease and PD. It has been shown, indeed, that 14-3-30 and 14-3$3 \eta$ can prevent the misfolding and aggregation of a-Synuclein both in vitro and in vivo, thus reducing its propagation and toxicity ${ }^{17,52,55}$. This may also explain why $14-3-3$ s are highly detected in LBs of PD patients ${ }^{56}$. This chaperone-like activity does 


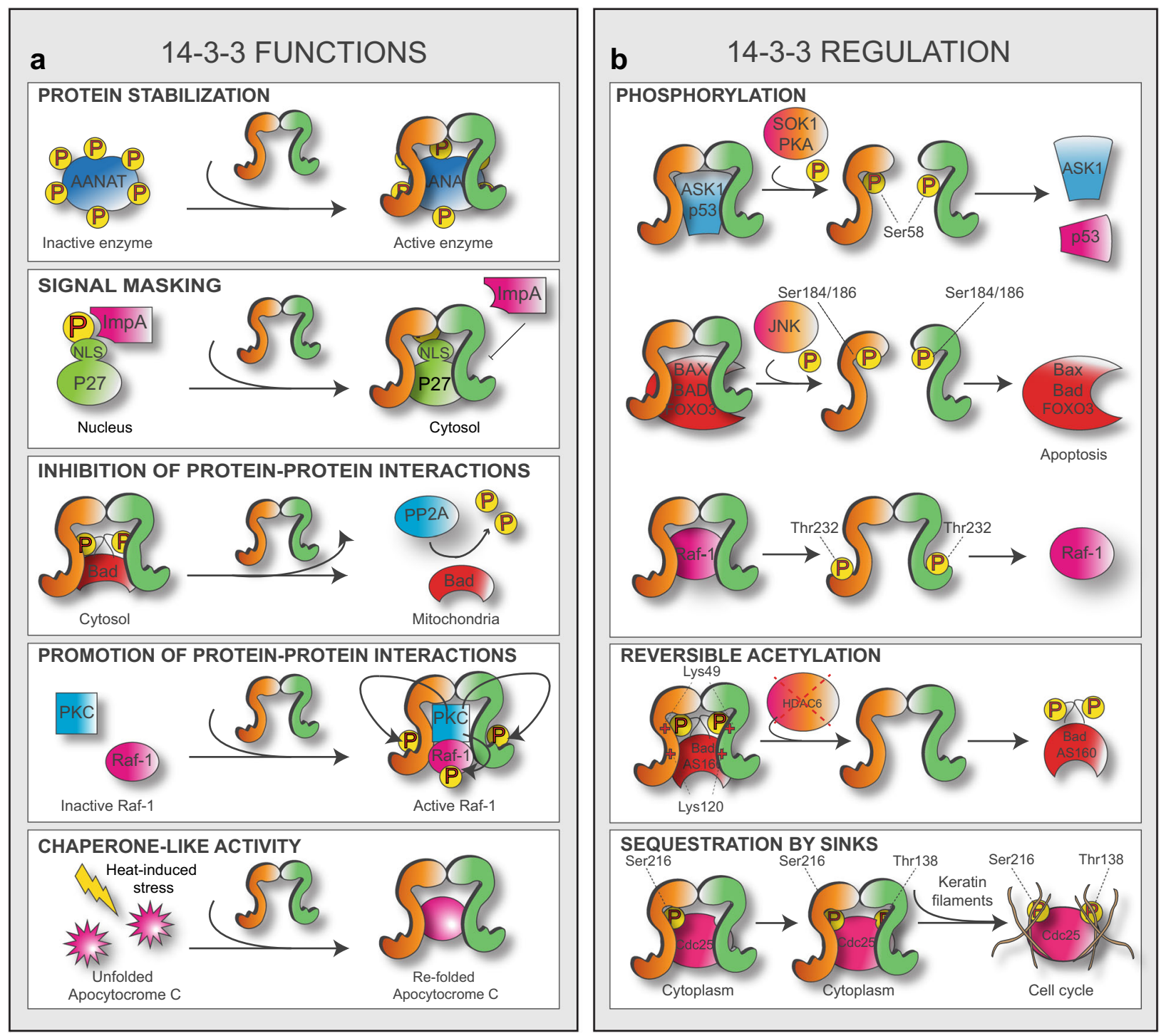

Fig. 2 Modifications and modalities of function of 14-3-3. Panels in $\mathbf{a}$ and $\mathbf{b}$ show some of the diverse modalities of activity and regulation of 14-3-3s as described in the main text. P: phosphate group; AANAT: serotonin N-acetyltransferase; ImpA: importin $\alpha$; NLS: nuclear localization signal; BAD: BCL2 associated agonist of cell death; PP2A: protein phosphatase 2; PKC: protein kinase C; Raf-1; ASK1: apoptosis signal-regulating kinase 1; SOK1; PKA: protein kinase A; BAX: BCL2 associated X; FOXO3: forkhead box class O 3; JNK: c-Jun N-terminal kinases; AS160: Akt substrate of $160 \mathrm{kDa}$; Cdc25.

not rely on the recognition and binding of specific phosphosequences, and it does not require the existence of either the hydrophobic groove or of the flexible C-terminal stretch ${ }^{57}$. Instead, a prominent role is exerted by the salt bridge interactions lying at the dimer interface, whose dynamic nature strongly impacts on the transient status of disorder at the $\mathrm{N}$-terminal region and on the monomer-dimer equilibrium of 14-3-3s. In particular, mutations of Asp21 or Glu89 are sufficient to alter the N-terminal ahelix order and shift the monomer-dimer balance in favor of the monomeric conformation. Similar to other chaperones, 14-3-3s must recognize and bind hydrophobic regions exposed by unfolded proteins. Therefore, by exposing some key residues, such as those framing the hydrophobic groove and the crucial Ser58 (see below), monomeric forms of 14-3-3s exhibit higher chaperone-like activities compared to their dimeric counterparts $26,34,58$. Nevertheless, evidence of phosphorylationdependent chaperone-like activity also exists. As an example, Liang and colleagues demonstrated that forskolin-induced phosphorylation of the R region of CFTR favors the binding of $14-3-3 \beta$ leading to an increase in CFTR expression and stability ${ }^{59}$.
Similar to heat shock proteins, 14-3-3 3 transcription is increased upon heat-induced stress in Drosophila, likely due to the presence of a heat-induced transactivator. This results in the dissolution of aggregated apocytochrome c and Hsp70/ Hsp40-mediated refolding of citrate synthase, once more suggesting relevant similarities between 14-3-3s and molecular chaperones (Fig. 2a, bottom box) $)^{60}$. The identification of the chaperone-like activity of $14-3-3$ s poses some new intriguing questions in the field. How does this role co-exist with the more canonical phospho-specific-targeting molecular hubs? What are the molecular cues that determine which activity 14-3-3s need to take on? How is chaperone-specificity achieved? Further experiments are needed to shed some light on these crucial questions. However, the observation that one single protein has been given custody of such a variegated repertoire of activities, might suggest a conservation process where modifications of a pre-existing abundant protein might have occurred cheaper and faster, in evolutionary terms, than the de novo creation of many different proteins. 


\section{Regulation of 14-3-3s}

The 14-3-3 interactome can be further enlarged by a variety of post-translational modifications (PTMs) (for relevant reviews see ref. ${ }^{61}$ and ref. ${ }^{62}$ ). One of the most common PTMs is phosphorylation. Accordingly, 14-3-3s contain a few critical Ser/ Thr residues whose phosphorylation can affect their dimerization status and interaction with target proteins. In particular, the invariant residue Ser $58 / 59$ of $14-3-3 \eta,-\gamma,-\beta$, and $-\zeta$ can be recognized by different kinases, leading, in the majority of the cases to 14-3-3s dissociation and reduced functional interaction with their associated partners (Fig. 1b). As an example, p21activated protein kinase 6 (PAK6), recognizes and phosphorylates Ser59 of 14-3-3y, thus promoting its dissociation from LRRK2 (see below) ${ }^{63}$. Intriguingly, in both $\sigma$ and $\theta / \tau$ isoforms, Ser58 is naturally replaced by an alanine residue, which, by definition, cannot be phosphorylated ${ }^{64}$. Doubts remain on how kinases can reach such a hidden spot, although sphingosine has been shown to bind 143-3s and change 14-3-3 conformation, thus rendering Ser58 prone to phosphorylation ${ }^{30,64}$

Other two residues that can be phosphorylated are Ser/Thr232 and Ser184/186. This latter was initially identified within a consensus motif for proline-directed kinases and believed to belong to $14-3-3 a$ and $14-3-3 \delta$, subsequently recognized to be the Ser184-phosphorylated forms of $14-3-3 \beta$ and $14-3-3 \zeta$, respectively ${ }^{19,65}$. Ser184/186 of $14-3-3 \zeta$ and $14-3-3 \sigma$ is a target of the c-Jun N-terminal kinase (JNK). Upon phosphorylation, 14-3-3Z and 14-3-3 $\sigma$ release the pro-apoptotic Bax, which then translocates to the mitochondria and initiates the apoptotic cascade ${ }^{66}$. Phosphorylation of Thr232 inhibits the binding between 14-3-3 3 and Raf$1^{67}$, likely the result of a conformational change of the C-terminal stretch, which is involved in 14-3-3:target binding and contains Thr232 (Fig. 2b, top box) ${ }^{68}$.

Acetylation of lysine residues is another relevant PTM observed on $14-3-3 s^{24}$. The addition of acetyl groups masks the positive charge of lysine residues, some of which are present in the binding groove and are crucial for the interaction with target proteins. Inhibition of histone deacetylase 6 (HDAC6) activity on K49 and K120 causes the dissociation of $14-3-3 \zeta$ from the AKTphosphorylated GTPase proteins AS160 and BAD, an event that is prevented in mutant forms of $14-3-3 \zeta$ in which these lysine residues have been replaced by arginine (Fig. 2 b, middle box) ${ }^{69,70}$. The shift between lysine acetylation and deacetylation may represent another dynamic mechanism to regulate the status of the 14-3-3 interactome ${ }^{70}$.

An additional way to regulate $14-3-3$ s is gene methylation. Many studies have demonstrated a link between hypermethylated CpG-rich regions of $14-3-3 \sigma$ and cancer $^{71}$. This epigenetic modification leads to a decrease in 14-3-3 $\sigma$ expression and has been observed in a variety of cancers, including breast cancer ${ }^{72}$, hepatocellular carcinoma ${ }^{73}$, and squamous cell carcinoma ${ }^{74}$. The in vitro treatment of cancer cells with $5^{\prime}$-aza-2'-deoxycytidine (an inhibitor of DNA methylation) recovers $14-3-3 \sigma$ expression $^{72,75}$.

Another way to regulate 14-3-3s functionality is offered by "sinks", partners which work as molecular magnets to capture 143-3s involved in loose interactions and commit them to new target proteins (Fig. 2b, bottom box) ${ }^{62,76-79}$.

The existence of such a wide potential of mechanisms to regulate 14-3-3 interactions with their target proteins provides further emphasis on the dynamic activity of 14-3-3s, which is crucial to their biological role.

\section{4-3-3 roles in the brain}

14-3-3s were originally described as abundant proteins in the brain (up to $1 \%$ of total soluble proteins, and their presence in both neurons and glia has been extensively investigated ${ }^{80-82}$.

14-3-3s are involved in several aspects of brain development, such as neurogenesis, neuronal differentiation and migration, neurite outgrowth, and axonal guidance ${ }^{83-85} \cdot 14-3-3$ s take part also in the shaping and maintenance of dendritic spines ${ }^{86,87}$, synaptic plasticity ${ }^{88}$, and neurotransmitter release ${ }^{89}$. In particular, $-\varepsilon$ and $-\zeta$ isoforms are critical in the early stages of brain development. $14-3-3 \varepsilon$ binds to and protects the phosphorylated form of nuclear distribution gene E-like homolog 1 (NDEL1). In turn, NDEL1 binds to Lissencephaly-1 (LIS1) and dynein to promote dynein motor function and neuronal migration (Fig. 3a, top left box). The absence of $14-3-3 \varepsilon$ exposes NDEL1 to the action of protein phosphatase $2 \mathrm{~A}$ (PP2A), thus impairing the migration process ${ }^{90}$. 14-3-3ع KO and $14-3-3 \varepsilon / \zeta$ double KO mice show severe deficits in cortical layer formation and suffer a significant rate of embryonic lethality. Likewise, deletions in the gene coding for $14-3-3 \varepsilon$ in humans are responsible for the onset of Miller-Dieker Syndrome, a severe form of Lissencephaly ${ }^{91,92}$. Similarly, lack of 143-3 affects the structure of the Disrupted-in-Schizophrenia 1 (DISC1)/NDEL1/LIS1 complex, resulting in migration defects and abnormal synaptic connectivity of hippocampal mossy fibers. In agreement, 14-3-3 $\zeta$ knockdown mice develop cognitive and behavioral impairments resembling those observed in schizophrenic patients ${ }^{93}$. Moreover, the 14-3-3 functional KO mouse, which expresses the pan 14-3-3 competitive peptide inhibitor difopein (dimeric fourteen-three-three peptide inhibitor), shows similar cognitive and behavioral deficits associated with impaired hippocampal long-term potentiation ${ }^{87}$.

14-3-3s influence growth cone morphology by acting on molecular pathways that transfer extracellular cues to cytoskeletal proteins ${ }^{94}$. Several 14-3-3 isoforms are expressed in the developing dorsal root ganglia in chickens and rats, where they affect the direction of the growing axon. As an example, 14-3-3s stabilize PKA catalytic subunits, preventing the phosphorylation of their downstream targets. This results in a different response (repulsion vs attraction) to nerve growth factor gradients (Fig. 3a, middle left box $)^{95}$. 14-3-3s are also involved in axon regeneration as shown with in vitro experiments of axonal damage, and these activities can be controlled with the use of Fusicoccin-A (FC-A). FC-A stabilizes the binding between 14-3-3s and general control nonderepressible 1 (GCN1) to regulate its degradation and promote neurite outgrowth. FC-A also prevents the die-back phenomenon observed in mice upon dorsal hemisection of the spinal cord ${ }^{96} .14-$ 3-3s also interact with the phosphorylated Tricornered-dependent kinesin Pavarotti in Drosophila. This complex inhibits the microtubule sliding which is necessary for neurite outgrowth, once more suggesting the involvement of $14-3-3 \mathrm{~s}$ in dendritic elongation ${ }^{97}$. Other studies using the functional 14-3-3 KO mouse have confirmed a role for $14-3-3$ s in regulating dendritic length and complexity ${ }^{87,98}$. This process involves several mechanisms, including modulation of the cell adhesion molecules L1 and neural cell adhesion molecule, actin-depolymerizing factor, neuron navigator 2, SLITRK-like family member 1, and the RafMEK-ERK pathway (Fig. 3a, bottom left box) ${ }^{94,99-103}$.

14-3-3s are highly expressed at synaptic terminals, where they bind phosphorylated target proteins of the presynaptic zone, such as Bassoon or $\mathrm{Ca}(\mathrm{V}) 2.2$ channel subunits and contribute to synaptic plasticity and transmission (Fig. 3a, top right box) ${ }^{86,104}$. 14-3-3s interact with the glutamate transporter EAAT2/GLT-1, which is in charge of glutamate reuptake at synapses ${ }^{105}$. Notably, GLT-1 expression and glutamate homeostasis are impaired in several models of $\mathrm{PD}^{106}$. 14-3-3s also affect the expression of several glutamate receptor subunits, thus suggesting a role in post-synaptic transmission ${ }^{107-109}$.

Moreover, 14-3-3s bind and activate tyrosine hydroxylase (TH), the step-limiting enzyme required for the biosynthesis of catecholamines, including dopamine ${ }^{4}$. TH can be regulated at several levels, including protein synthesis, mRNA stability, and activity. TH harbors three different Ser residues (Ser19, Ser31, Ser40) whose phosphorylation is essential to its functionality. In 


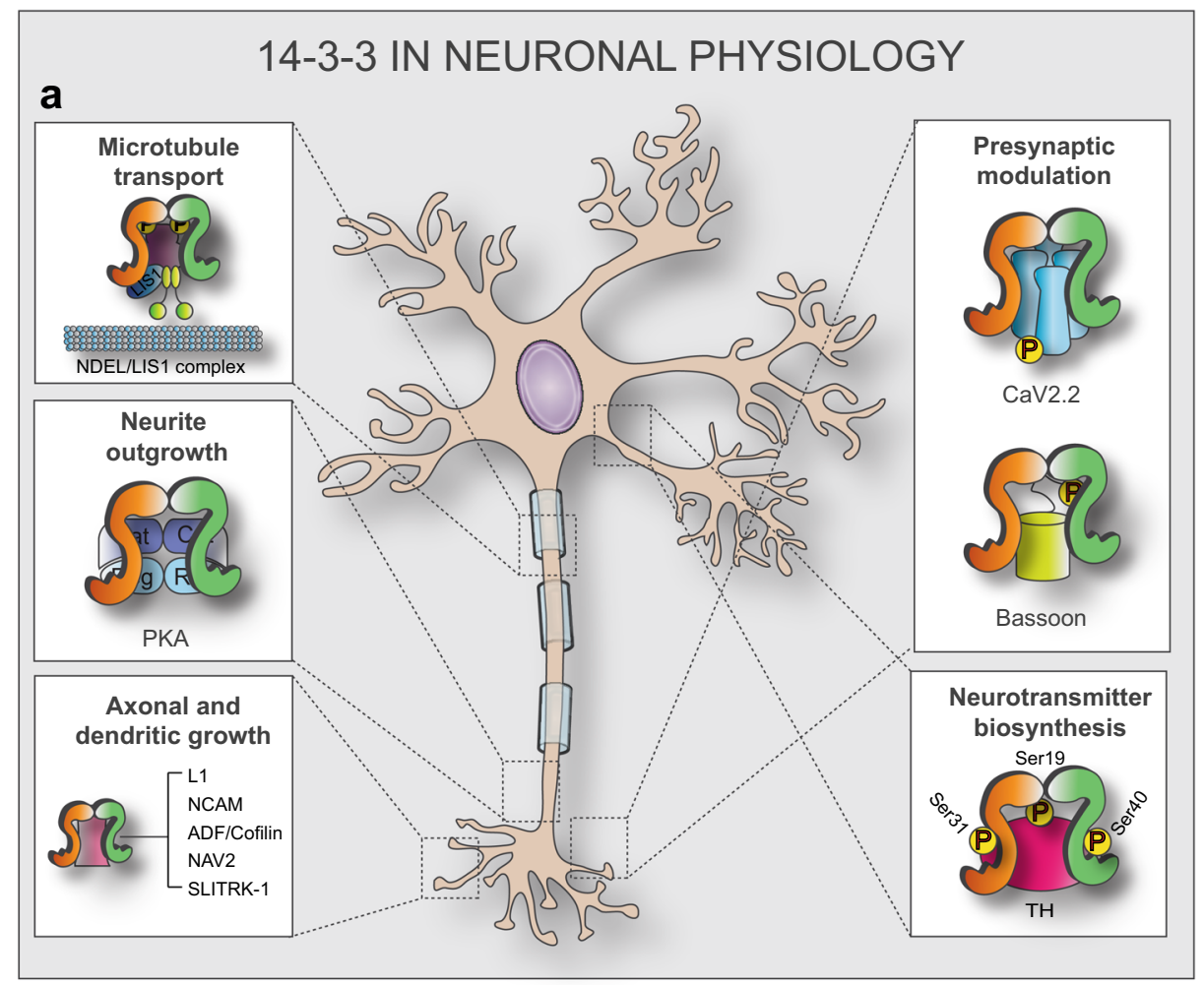

\section{b}

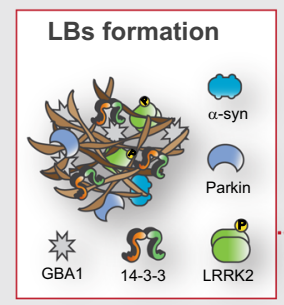

14-3-3 IN PD PATHOGENESIS

LRRK2-mediated toxicity

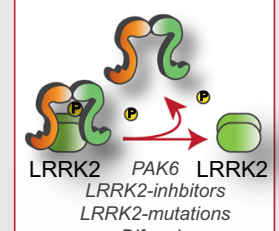
Difopein

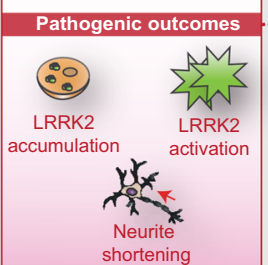

shortening

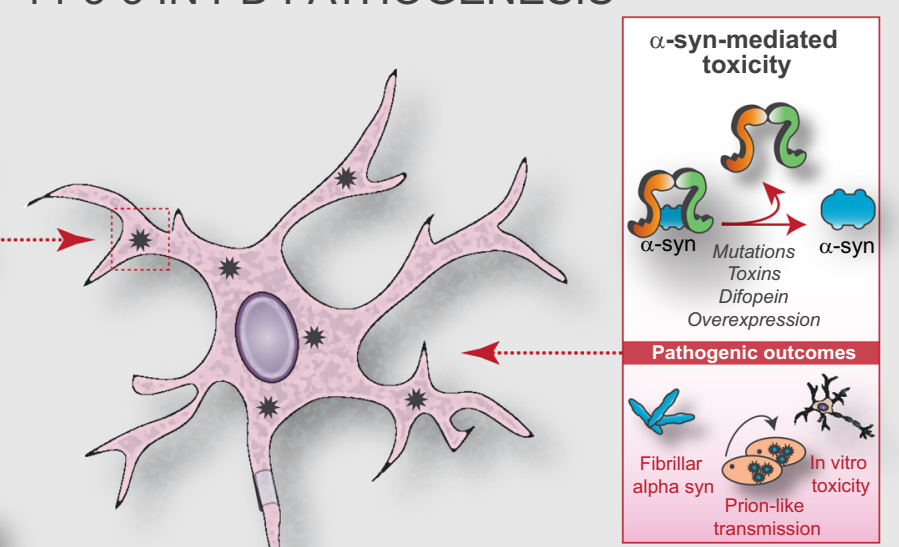

$$
\text { transmission }
$$

Parkin-mediated clearance

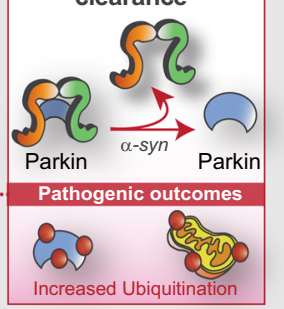

Fig. 3 Roles of 14-3-3 in the healthy and PD brain. The roles of 14-3-3s in physiological conditions (a) and its involvement in PD (b). a NDEL/ LIS: nuclear distribution gene E-like homolog 1/Lissencephaly-1; PKA: protein kinase A; NCAM: neural cell adhesion molecule; ADF/Cofilin: actin-depolymerizing factor/Cofilin; NAV2: neuron navigator 2; SLITRK-1: SLIT and NTRK-like family, member 1; CaV2.2: N-type voltage-gated $\mathrm{Ca}^{+}$channel; Bassoon; TH: tyrosine hydroxylase. b GBA: glucosylceramidase beta; LRRK2: leucine-rich repeat kinase 2; $\alpha$-Syn: $\alpha-S y n u c l e i n ;$ PAK6: P21-activated kinase 6.

particular, 14-3-3s bind $\mathrm{TH}$ at phosphorylated Ser19. This interaction allows $14-3-3 \mathrm{~s}$ to stabilize $\mathrm{TH}$ and assist in the regulation of both Ser31 and Ser40 phosphorylation (Fig. 3a, bottom right box $)^{110}$.
All together, these observations indicate a significant role of 14$3-3 \mathrm{~s}$ in the development and function of the central nervous system. It is therefore not surprising that 14-3-3s may also be linked to the pathogenesis of several neurological diseases. 


\section{4-3-3 roles in Parkinson's disease}

The progressive degeneration of dopaminergic neurons in the substantia nigra pars compacta, results in a reduction in dopamine, which is a key neuropathological feature of $P D^{111-113}$. Depletion of striatal dopamine underlies the classic motor symptoms observed in PD, such as bradykinesia, rest tremor, rigidity, and postural instability $^{113}$. Several experimental models have been generated to recapitulate these neuropathological conditions. Among these, the most used are animals treated with toxins that disrupt the nigrostriatal pathway (e.g., 6-hydroxydopamine), inhibitors of the mitochondrial complex I (e.g., 1-methyl-4-phenyl-1,2,3,6-tetrahydropyridine and rotenone), and proteasomal inhibitors (e.g., epoximycin) ${ }^{114}$. Interestingly, about $10 \%$ of total PD cases are familial, and several genes are known to be mutated in dominant or recessive forms of PD. Among these, mutations in SNCA and LRRK2 (encoding a-Synuclein (a-Syn) and LRRK2), cause autosomal dominant forms of PD closely resembling sporadic PD, while recessive mutations in PRKN, PTEN-induced kinase 1, and PARK7 genes (encoding for parkin, PINK-1, and DJ-1) cause early-onset parkinsonism ${ }^{115}$. Additional genome-wide association studies have pointed to single-nucleotide polymorphism within $L R R K 2$, $S N C A$, and GBA1, encoding the lysosomal glucocerebrosidase, as risk factors for the development of $P D^{116}$. The discovery of mutations in specific genes contributing to the pathology of PD has led to the development of transgenic models, which recapitulate certain but not all the pathophysiological features present in PD patients ${ }^{117}$.

Brains of PD patients demonstrate Lewy bodies (LBs) and Lewy neurites, abnormal insoluble aggregates containing fibrillar proteins found in the substantia nigra, locus coeruleus, and other brainstem regions along with regions of the cerebral cortex ${ }^{118,119}$. a-Syn, together with ubiquitin, is one of the major protein components of $\mathrm{LBs}^{120}$. Recent in-depth ultrastructural analyses have shown that LBs are composed of membranous material originating from vesicles and fragmented organelles including mitochondria, lipids, and lysosomal structures ${ }^{121}$. Moreover, LBs contain a mixture of proteins (including TH, DJ-1, LRRK2, Parkin, PINK-1, GBA1, SPG11/spatacsin ${ }^{122}$, and mitochondria-related proteins) and molecules implicated in the ubiquitin-proteasome system, autophagy, and aggresome formation (reviewed in ref. ${ }^{123}$ ). Importantly, $14-3-3$ s are present in LBs and are known to interact and co-localize with several proteins involved in PD pathogenesis, including LRRK2, a-Syn, and parkin (Fig. 3b, top left box) ${ }^{124}$ or in other neurodegenerative disorders with parkinsonian syndrome such as hereditary spastic paraplegia caused by mutations in $S P G 11^{125}$. To this regard, we recently observed that PKA-mediated phosphorylation of Ser955 in SPG11/spatacsin is a signal for the binding of 14-3-3n (https://doi.org/10.1101/ 2020.09.09.289009 ${ }^{126}$ ). Whether 14-3-3 dysregulation is a cause or a consequence of LB formation, is still not clear.

Overexpression of $14-3-3 \theta / \tau,-\varepsilon$, and $-\gamma$ is protective against neurotoxin models, such as rotenone and MPTP, while difopein exacerbates cell death ${ }^{16}$. Similar results have been obtained in vivo, where $14-3-3 \theta / \tau$ protects surviving dopaminergic neurons in a model of MPTP-induced toxicity ${ }^{127}$. Neuroprotective effects are instead lost when $14-3-3 \theta / \tau$ is phosphorylated at Ser232 in neuroblastoma cells, most likely because of the inability of the phosphorylated isoform to inhibit Bax and its downstream targets $^{128,129}$. Of note, $14-3-3 \theta / \tau$ phosphorylated at Ser232 is increased on the insoluble fraction of brain lysates from PD patients ${ }^{129,130}$. Thus, 14-3-3s co-localization in LBs, their altered phosphorylation in PD models and patients, and their acknowledged neuroprotective effects in several disease models leads to the speculation that $14-3-3 \mathrm{~s}$ are likely to play a crucial role in the pathophysiology of $\mathrm{PD}$, and their regulation may represent a possible strategy for future therapies.
Interaction between 14-3-3s and LRRK2. LRRK2 is a large multidomain protein belonging to the ROCO family. It includes a characteristic bimodal region composed of a Ras Of Complex (ROC) domain, responsible for GTP binding and hydrolysis, and a C-terminal Of ROC (COR) domain, which works as a dimerization hub $^{131}$. The ROC-COR tandem is followed by a serine/threonine kinase domain regulated by the GTPase activity, implying a complex intramolecular control ${ }^{132,133}$. Several repeats regions flank this tripartite enzymatic core in the following order: armadillo (ARM), ankyrin (ANK), and leucine-rich repeats (LRR) at the $\mathrm{N}$ terminal, and WD40 repeats at the C-terminal, collectively involved in the regulation and localization of LRRK2 ${ }^{134}$. Several missense mutations clustered in the catalytic core $(\mathrm{N} 1437 \mathrm{H}, \mathrm{R} 1441 \mathrm{C} / \mathrm{G} / \mathrm{H}$, Y1699C, G2019S, and I2020T among others) are pathogenic with age-dependent penetrance. Together, these mutations cause autosomal dominant forms of PD that account for $4 \%$ of familial and $1 \%$ of sporadic cases, although these percentages may increase depending on the population (for a detailed review, please refer to ${ }^{135}$ ). Among these, the G2019S mutation is the most frequently found in familiar PD and results in clinical and pathological features that closely resemble those described for idiopathic patients, including the presence of LBs and the loss of midbrain dopaminergic neurons ${ }^{136}$. In most cases, these mutations alter the biochemical activities of LRRK2. For example, G2019S and I2020T, both located at the kinase domain, increase the phosphorylation activity of LRRK2 $2^{137,138}$, most likely through the stabilization of its active status ${ }^{139}$. Alternatively, mutations at the ROC-COR domain have a direct effect on ROC by slowing GTP hydrolysis, which affects cellular phosphorylation of LRRK2 substrates by impairing LRRK2 recruitment to signaling compartments or by improper interactions with ROC binding partners ${ }^{132,140-143}$.

LRRK2 sequence includes several phosphorylation sites, some of which (e.g., Ser1292) are LRRK2 autophosphorylation sites and can be used as bona fide functional readouts to monitor its kinase activity ${ }^{144}$. This is remarkably important, considering that the altered phosphorylation of LRRK2 substrates strongly associates with the pathobiology of PD both in experimental and clinical settings ${ }^{145,146}$. Furthermore, the phosphorylation status of LRRK2 crucially influences its interactions with other proteins, among which are 14-3-3s. Several 14-3-3-binding sites, where LRRK2 is constitutively phosphorylated, are spread across the entire structure of the protein, and include Ser860 on the ANK domain, Ser910, Ser935, Ser955, and Ser973 at the N-terminal, Ser1444 within the ROC domain, and the latest identified Thr2524 at the C-terminus ${ }^{14,147}$. In a very interesting work, Stevers et al. examined the binding affinity and crystal structure of 14-3-3y complexed with different LRRK2 peptides, each containing regions around the phosphorylation sites Ser860, Ser910, Ser935, Ser955, Ser973, and Ser1444. In agreement with previous results obtained by the same and other labs for c-Raf, PKC, and CFTR ${ }^{148-151}$, simultaneous interactions of $14-3-3 \gamma$ with multiple docking sites within LRRK2 results into synergistic affinity values. In particular, interactions obtained by combining peptides containing two phosphorylation sites selected among Ser910, Ser935, and Ser1444 attained nanomolar affinity values. As lately outlined by Sluchanko in ref. ${ }^{152}$, the presence of multivalent sites poses a spiny question related to the stoichiometry of 14-3-3 complexes, which is further amplified in the case of large proteins, such as LRRK2, for which a complete structural analysis in complex with $14-3-3$ s is still missing. In a recent comparative analysis, Manschwetus et al. investigated the binding affinity shown by each 14-3-3 isoform and confirmed that all but the $\sigma$-isoform are able to bind LRRK2, with the highest affinity shown by isoforms $-\gamma$ and $-\eta^{14}$. We also showed that $14-3-3 \gamma$ is able to bind LRRK2. Upon phosphorylation at Ser59 by PAK6, 143-3y monomerizes and dissociates from LRRK2, which, in turn, becomes dephosphorylated at Ser935. Notably, with this mechanism, PAK6 is able to rescue neurite shortening caused by G2019SLRRK2 in primary neurons ${ }^{63}$. 
Ser910/935 are not sites of autophosphorylation, as shown by the fact that kinase-dead variants of LRRK2 only lead to a partial dephosphorylation of these residues and/or LRRK2 relocalization ${ }^{153}$. This implies that alternative kinases, such as PKA ${ }^{154}$, inhibitor of nuclear factor $K B(I K B)^{155}$, and casein kinase I alpha (CKla) ${ }^{156}$ may be involved. Phosphorylation at these sites is compromised in several mutant forms of LRRK2 associated with PD. For example, R1441G and Y1699C, and to a minor extent G2019S, show reduced phosphorylation of Ser935 and concomitant impairment of 14-3-3 binding ${ }^{154}$. Similarly, mutations at R1441 prevent PKA phosphorylation of Ser1444, and with this, the binding of 14-3-3s. Curiously, LRRK2 inhibitors also induce LRRK2 dephosphorylation at Ser910/935 and consequent 14-3-3 release. One possibility is that LRRK2 inhibitors destabilize LRRK2, leading to a reduced amount of the protein and thus explaining their beneficial effects ${ }^{157}$. The pathological reduced binding of LRRK2 to 14-3-3s may lead to different functional outcomes, such as an increased activity of LRRK2 $2^{98,158}$, re-localization, and accumulation of LRRK2 into cytoplasmic pools reminiscent of those observed in PD patients ${ }^{147}$, or the exclusion of LRRK2 release in exosomes (Fig. 3b, bottom left box) ${ }^{159}$. Indeed, PD rodent models and postmortem PD brains show reduced 14-3-3: LRRK2 interaction and an associated increase in LRRK2 kinase activity $^{160}$. As mentioned, dephosphorylation of Ser910/935 induced by LRRK2 inhibitors is also associated with increased ubiquitination and partial degradation of LRRK2. In accordance, R1441C, Y1699C, and I2020T mutants show dephosphorylation at Ser935, cytoplasmic accumulation, and increased LRRK2 ubiquitination $^{161}$. Also, dissociation of the 14-3-3:LRRK2 complex and re-localization of LRRK2 in the cytoplasmic clusters occurs upon PAK6-dependent phosphorylation of 14-3-3y (unpublished observations). In accordance, overexpression of $14-3-3 \theta / \tau$ and direct binding with LRRK2 reduce its kinase activity and reverse neurite shortening observed in G2019S-LRRK2 transgenic mice, while $14-3-3 \theta / \tau$ inhibition with the competitive peptide inhibitor difopein disrupts its binding to LRRK2, promotes LRRK2 kinase activity, and further increases neurite shortening in G2019SLRRK2 $^{\text {mice }}{ }^{98}$. This suggests that the binding of 14-3-3s to LRRK2 may induce a conformational change, shifting the kinase into its inactive form ${ }^{158}$. Consistent with this, LRRK2 inhibitors reverse the neurite shortening induced by difopein, suggesting that the regulation of kinase activity by $14-3-3 \mathrm{~s}$ is required for this protective effect on neurite dynamics ${ }^{98}$.

Interaction between 14-3-3s and a-Synuclein. Together with isoforms $-\beta$ and $-\gamma$, $\alpha$-Syn defines the family of synucleins in mammals. a-Syn is a small $(14.4 \mathrm{kDa})$, intrinsically disordered protein mainly localized at the presynaptic terminals and involved in the regulation of synaptic function and plasticity ${ }^{162}$. So far, five rare autosomal dominant mutations (A30P, E46K, H50Q, G51D, and A53T) as well as genomic copy number variations have been identified and associated with the pathogenesis of PD, suggesting that alterations in the gene may be causally related to neuronal degeneration and LB formation ${ }^{163-169}$. a-Syn contains 4 tyrosine residues (Tyr39, Tyr125, Tyr133, and Tyr136) that can be crosslinked by oxidants and nitrating agents and form insoluble aggregates ${ }^{170}$. In animal models, the overexpression of a-Syn or the injection of a-Syn fibril seeds leads to cellular and tissue alterations reminiscent of those observed in PD patients, such as loss of dopaminergic neurons, neurite swelling, and activation of microglia and peripheral inflammatory cells ${ }^{171-173}$. a-Syn and 143-3s share regions of homology, and a-Syn interacts with some targets of 14-3-3s, such as BAD and PKC, although with differences in affinity and subcellular localization ${ }^{174}$. a-Syn has one major site of phosphorylation, Ser129, which is partially phosphorylated in basal conditions ${ }^{175}$, and one additional phosphorylation site, Ser87, which seems to be specific to the a-isoform ${ }^{176}$. Both of these sites can be phosphorylated by casein kinases CK1 and CK2 as well as polo-like kinase 2 (PLK2), while Tyr125 can be substrate of Src kinases ${ }^{176,177}$. Phosphorylation at Ser129 is drastically and selectively increased in the brains of patients affected by synucleinopathies and is faithfully mirrored in several animal models ${ }^{178,179}$. Phosphorylation at this residue works as a molecular switch to regulate a-Syn activity and subcellular localization as well as its binding to membranes, ions, and proteins ${ }^{180}$. LBs are enriched in a-Syn phosphorylated at Ser129, in both humans and experimental models ${ }^{178}$. Of note, the phosphorylation of a-Syn is not a prerequisite for 14-3-3 binding. Indeed, low stoichiometric amounts of 14-3-3n can interact with unphosphorylated $a-S y n$ and reduce the formation of toxic a-Syn aggregates in vitro ${ }^{17}$. This is in agreement with studies suggesting that $14-3-3 \theta / \tau$ may act as a chaperone and protect a-Syn from folding into a pathogenic conformation or even reshape already misfolded a-Syn. In vitro and cellular experiments have shown that $14-3-3$ isoforms $-\varepsilon,-\gamma$, and $-\theta / \tau$ reduce $a-S y n$ inclusions and

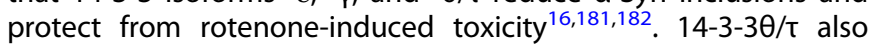
reduces a-Syn aggregation and neuronal loss in cellular and in vivo fibril models, while difopein accelerates a-Syn aggregation and toxicity ${ }^{52}$ and $^{55}$.

a-Syn possesses prion-like behavior and can therefore transfer from one cell to another through tunneling nanotubes, exosomes, or other cellular processes ${ }^{183}$. This prion-like behavior occurs also in PD patients, in which healthy neuronal transplants show hostderived misfolded a-Syn a few years after the $\operatorname{graft}^{184,185}$. This physical transmission may explain the spreading of a-Syn to different brain regions over time, which remarkably overlaps with the progression of clinical symptoms in PD patients, as postulated by Braak and colleagues ${ }^{186-188}$. 14-3-30/ may impede cell-to-cell transmission of pathologic a-Syn, as demonstrated by studies using paracrine cellular models. Despite promoting total $a-S y n$ release, overexpression of $14-3-3 \theta / \tau$ reduces the release of misfolded a-Syn species, thus preventing its diffusion and internalization by surrounding neurons (Fig. 3b, top right box). Similarly, manipulation of 14-3-3s also alters the propagation of aSyn across synapses in the fibril model, as determined by the use of multi-chamber, microfluidic devices ${ }^{52}$.

What leads to 14-3-3 dysfunction in PD is unclear. One possibility is that excess a-Syn may promote 14-3-3 dysregulation, and different hypotheses have been formulated on the possible mechanisms. a-Syn might act at the transcriptional level, as supported by the observation that $14-3-3$ isoforms $-\varepsilon,-\gamma$, and $-\theta / \tau$ are downregulated in cells and mice overexpressing $a-S y n{ }^{16,127}$. Also, by interacting with 14-3-3s, a-Syn could sequester 14-3-3s and reduce its inhibitory action on pro-apoptotic partners, such as $B A D$, thus leading to neuronal vulnerability ${ }^{129,189}$. Soluble $a-S y n$ complexes may induce specific dopamine toxicity through the generation of reactive oxygen species and the withdrawal of 14-3$3 s^{189}$.

Reduction in functional 14-3-3 levels by either transcriptional dysregulation or sequestration would thereby limit the ability of 14-3-3s to regulate the activity of its partners, including a-Syn and LRRK2. In addition, a-Syn overexpression increases 14-3-30/ phosphorylation at Ser232 in several PD models, and increased Ser232 phosphorylation is observed in insoluble fractions from postmortem human PD brains ${ }^{128,130}$. Ser232 phosphorylation is associated with a loss of $14-3-3 \theta / \tau^{\prime}$ s neuroprotective effects against rotenone and MPTP ${ }^{128}$. Aberrant Ser232 phosphorylation could potentially disrupt the interaction of $14-3-3 \theta / \tau$ with a-Syn and LRRK2, and thereby lead to toxicity due to increased a-Syn misfolding and LRRK2 kinase activity.

Interaction between 14-3-3s and Parkin. Parkin is a $52 \mathrm{kDa}$ E3 ubiquitin-protein ligase involved in several cellular pathways, including the degradation of misfolded proteins through the ubiquitin-proteasome system and the regulation of mitochondrial homeostasis $^{190}$. Parkin structure is characterized by a sequential 
string of domains: a ubiquitin-like domain (Ubl) at its $\mathrm{N}$ terminus followed by two zinc-coordinating RING (really interesting new gene)-like domains (RING0, RING1), one IBR (in between RING finger) domain, a linker region, and a final RING2 domain ${ }^{191}$. Several mutations of Parkin have been associated both with autosomal recessive early-onset parkinsonism, usually characterized by the absence of LB formation, and with sporadic forms of PD ${ }^{192-194}$. Parkin interacts with a-Syn and participates in many pathways associated with its regulation, likely contributing to the degradation of the misfolded protein and the rescue of the toxic effects $^{195,196}$. Parkin also associates and co-localizes with 14-3-3n in LBs. This interaction is mediated by the linker region of Parkin, resulting in the inhibition of Parkin self-ubiquitination as well as the ubiquitination of its cellular targets (Fig. 3b, bottom right box). Curiously, the wild-type form of a-Syn (but not the mutant A30P or A53T) can disrupt the binding between 14-3-3n and Parkin by sequestering $14-3-3 \eta^{15}$. Moreover, Parkin and 14-3-3s are both involved in the removal of damaged mitochondria, a process called mitophagy. Under autophagy-inducing stress conditions, Parkin ubiquitinates proteins in the outer membrane of mitochondria. This begins a cascade of events that culminate with the lack of mammalian target of rapamycin complex 1 (mTORC)-dependent phosphorylation of the transcription factor EB (TFEB). Unphosphorylated TFEB cannot bind to cytoplasmic $14-3-3$ s and is therefore translocated to the nucleus where it induces the transcription of different lysosomal and autophagic genes ${ }^{197,198}$.

All together, these observations suggest that a better understanding of the regulatory pathways controlling the interactions of 14-3-3s with LRRK2, a-Syn, and Parkin will be crucial to get further insights into the pathogenesis of PD and may serve as a potential target in the search for new therapeutic strategies.

\section{Stabilizers and inhibitors of 14-3-3s: translational opportunities?}

Given the large amount of client proteins and the consequent variety of cellular activities involved, it is reasonable to consider 14-3-3s as prospective targets for future drug therapies.

The first step in the development of new drugs relies on the identification of candidate targets. In this context, computational algorithms based on position-specific scoring matrix, support vector machine, or artificial neural networks, such as Scansite 4.0, EML, NetPhorest, ANIA, and 14-3-3 Pred, are now available to the community and have become of use to predict sequence-specific binding motifs ${ }^{199-204}$. Once identified, these targets and their interaction with the protein of interest need to be validated. NMR and crystallographic data have provided major insights about the spatial conformation of 14-3-3 isoforms and their target-bound structures ${ }^{31}$. Although technical limitations have restricted the analyses mainly to $14-3-3 \mathrm{~s}$ associated with short peptide fragments, these have been critical in determining the physicalchemical principles ruling 14-3-3 PPIs. Even more, it is on the basis of these crystallographic analyses that the first small molecules have been identified and successively optimized ${ }^{205}$. More recently, the advent of computational algorithms has assigned new boundaries to our prediction potential. As mentioned, Stevers and coworkers developed a thermodynamic model to estimate the binding affinities of peptides derived from the multiple 14-3-3binding sequences of CFTR and LRRK2. In particular, with this model they were able to predict how the mutation/deletion of specific phospho-sites (e.g., pSer910 or pSer935 in LRRK2) could have affected the binding of the mentioned proteins to $14-3-3 s^{42}$. Models like this combined with classical crystal structural analyses will accelerate the identification and targeting of key residues whose modification might increase the efficacy and specificity of therapeutic small molecules. While specificity is always a soughtafter requirement in drug development, this is particularly trueand difficult to achieve-in the case of hot-spot proteins, such as 14-3-3s. Indeed, because of the high structural conservation of the binding groove and the occurrence of similar binding motifs in hundreds of target proteins, the risk of incurring unwanted side effects is potentially high. As mentioned, however, the specificity of the target binding does not depend on the conserved amphipathic groove, but it rather relies on the surrounding client-specific residues. Based on this observation, secondary binding sites can be pinpointed and specifically targeted to modulate $14-3-3$ s interactome ${ }^{206}$. While in some cases (e.g., 14-33:LRRK2 or 14-3-3:a-Syn) a stabilization of the 14-3-3:target binding is desirable, in others a de-stabilization would be preferred, locking the choice to the specific context. In both cases, PPI modulators may work either as competitors-contending the same binding spot with all the proteins that possess a region with similar structural features- or as allosteric modulators - changing the affinity of a protein for its binding partner in a more indirect way. Each of these solutions comes with pros and cons. Indeed, while competitive inhibitors are geared toward rational design strategies, they lack specificity, since they might block the interaction of the protein of interest (e.g., 14-3-3) with any of its partners. On the opposite, PPI stabilizers may be more difficult to frame, but they do ensure more specificity, since their glue-like action relies on the exclusive interaction platform offered by two interacting partners ${ }^{207-209}$.

So far, some promising results have been obtained by small molecules acting either as competitive inhibitors (e.g., R18, difopein) or as non-competitive stabilizers (fusicoccanes) of 14-33:target binding. R18 has been initially isolated from a phage display library; it can enter in the groove and affect the binding of the associated target ${ }^{210}$. As an example, R18 can inhibit the binding of $14-3-3$ s with Raf-1 or FOXO3a, ultimately leading to cell apoptosis and suggesting a possible strategy in the treatment of cancer ${ }^{210,211}$. As mentioned, R18 interferes in the interaction between 14-3-3s and PKA, leading to the dissociation of its regulatory and catalytic subunits ${ }^{95}$. Similarly to R18, difopein (composed by two repeats of the R18 peptide separated by a flexible linker) may induce apoptosis ${ }^{212,213}$. Interestingly, difopein has been used to inhibit the binding between 14-3-3s and LRRK2, proving that this interaction is necessary to LRRK2 activity ${ }^{98}$ and to prevent LRRK2 ubiquitination ${ }^{161}$. However, these peptides are non-specific, since they bind to the groove of all the isoforms and regardless of the associated target. Nevertheless, these first approaches proved that 14-3-3s may represent a druggable target and paved the way to the discovery of an increasing number of 14-3-3 PPI inhibitors with better specificity (for an extensive review, see ref. ${ }^{208}$ ).

Fusicoccanes are a family of natural small molecules that recognize the binding groove and stabilize 14-3-3:client complexes. However, their accessibility is constrained by specific residues in the binding motif, thus restricting their activities to a limited range of 14-3-3 targets $^{214}$. FC-A and the closely related Cotylenin $A$ (Cot-A) have been used to promote the interactions between 14-3-3s and some specific targets. FC-A can stabilize the interaction between $14-3-3 \beta$ and CFTR by selectively interacting with a secondary motif of CFTR. This strengthens the binding to 14-3-3 $\beta$ and promotes the transportation of CFTR ${ }^{151}$. FC-A also promotes neurite outgrowth in spinal cord and optic nerve injury models by stabilizing the interaction of $14-3-3 \mathrm{~s}$ with the stress response regulator GCN-196. Cot-A has been shown to work as an anti-tumor agent when used in combination with other molecules such as Vincristine or anti-EGFR antibody ${ }^{215}$. Cot-A selectively binds to the pSer233 and pSer259 residues of Raf, which are also putative 14-3-3 binding sites. The Raf:14-3-3 3 interaction at these Ser residues (but not to pSer621) results in Raf inhibition. The concomitant coupling of Cot-A further stabilizes this interaction and prevents Raf pro-survival activity ${ }^{216}$.

Interestingly, the presence of a C12-dehydroxy allows Cot-A to work as a stabilizer of target proteins binding either motif I or II. In 
contrast, hydroxylation occurring on FC-A prevents such interaction and classifies FC-A as a motif III-specific stabilizer ${ }^{208}$. These structural observations boosted the search for chemical modifications aimed at the development of semi-synthetic molecules endowed with functional specificity. Among these, FC-THF (modified with a tetrahydrofuran ring), ISIR-005 (which lacks C12 hydroxylation), and FC aglycones (where the glycosyl group has been replaced by a hydrogen) have been tested for their ability to stabilize 14-3-3:protein complexes ${ }^{217-219}$. High-throughput screenings of synthetic peptides have identified Epibestatin and Pyrrolidone- 1 as compounds able to specifically stabilize the interaction between $14-3-3 \mathrm{~s}$ and the proton pump PMA2, although their binding modality differs from each other ${ }^{220}$. Structural analysis identifying FC-A derivatives that increase neurite outgrowth revealed that these semi-synthetic FC-derived molecules have dual functionality to promote certain protein client interactions while disrupting others through structural shifts in the 14-3-3 binding pocket ${ }^{221}$.

All together these observations show that 14-3-3s could be legitimate candidates for drug discovery. In particular, the use of crystal structures and bioinformatics tools together with new technologies such as dynamic combinatorial chemistry, covalent tethering, and structure-activity relationship associated with NMR will greatly help the identification of new small molecules with enhanced therapeutic potential ${ }^{222-225}$.

\section{CONCLUSIONS}

Despite the lack of a signature catalytic activity, 14-3-3s are involved in a myriad of pathways critical to the maintenance of cellular homeostasis. By binding to specific phospho-motifs (although with some exception), 14-3-3s interact with a variety of intracellular proteins, including enzymes, transcription factors, and cytoskeletal proteins ${ }^{2}$. For this reason, 14-3-3s have acquired increasing interest over the years, boosted by evidence that altered 14-3-3 function is observed in numerous pathologies, such as cancer and neurodegenerative diseases, including PD. As a matter of fact, 14-3-3s interact with different proteins involved in the pathogenesis of PD, including LRRK2, a-Syn, and Parkin. Manipulation of 14-3-3:target protein interactions to interfere with some of the pathways involved in PD pathogenesis may serve as a new therapeutic approach. However, the search for drugs aimed at interfering with 14-3-3:target interaction must take into account the need for specificity. In this respect, the great advances made in the past decades in the field of X-ray crystallography, NMR spectrometry, and electron microscopy, as well as the implementation of databases and prediction algorithms, will be critical to exploit the full therapeutic potential of 14-3-3s.

Received: 4 March 2021; Accepted: 23 July 2021;

Published online: 21 September 2021

\section{REFERENCES}

1. Rosenquist, M., Sehnke, P., Ferl, R. J., Sommarin, M. \& Larsson, C. Evolution of the 14-3-3 protein family: does the large number of isoforms in multicellular organisms reflect functional specificity? J. Mol. Evol. 51, 446-458 (2000).

2. Dougherty, M. K. \& Morrison, D. K. Unlocking the code of 14-3-3. J. Cell Sci. 117, 1875-1884 (2004).

3. Paul, A. L., Denison, F. C., Schultz, E. R., Zupanska, A. K. \& Ferl, R. J. 14-3-3 phosphoprotein interaction networks-does isoform diversity present functional interaction specification? Front Plant Sci. 3, 190 (2012).

4. Ichimura, T., Isobe, T., Okuyama, T., Yamauchi, T. \& Fujisawa, H. Brain 14-3-3 protein is an activator protein that activates tryptophan 5-monooxygenase and tyrosine 3-monooxygenase in the presence of $\mathrm{Ca}^{2+}$, calmodulin-dependent protein kinase II. FEBS Lett. 219, 79-82 (1987).

5. Ford, J. C. et al. 14-3-3 protein homologs required for the DNA damage checkpoint in fission yeast. Science 265, 533-535 (1994).
6. Fantl, W. J. et al. Activation of Raf-1 by $14-3-3$ proteins. Nature $\mathbf{3 7 1}, 612-614$ (1994).

7. Freed, E., Symons, M., Macdonald, S. G., McCormick, F. \& Ruggieri, R. Binding of 14-3-3 proteins to the protein kinase Raf and effects on its activation. Science 265, 1713-1716 (1994).

8. Bonnefoy-Berard, N. et al. Inhibition of phosphatidylinositol 3-kinase activity by association with 14-3-3 proteins in T cells. Proc. Natl Acad. Sci. USA 92, 10142-10146 (1995).

9. Robinson, K. et al. Mechanism of inhibition of protein kinase $C$ by 14-3-3 isoforms. 14-3-3 isoforms do not have phospholipase A2 activity. Biochem J. 299, 853-861 (1994).

10. Morgan, A. \& Burgoyne, R. D. Exo1 and Exo2 proteins stimulate calciumdependent exocytosis in permeabilized adrenal chromaffin cells. Nature 355, 833-836 (1992).

11. Muslin, A. J., Tanner, J. W., Allen, P. M. \& Shaw, A. S. Interaction of 14-3-3 with signaling proteins is mediated by the recognition of phosphoserine. Cell $\mathbf{8 4}$, 889-897 (1996).

12. Henriksson, M. L. et al. A nonphosphorylated 14-3-3 binding motif on exoenzyme $S$ that is functional in vivo. Eur. J. Biochem. 269, 4921-4929 (2002).

13. Ulitsky, I., Krishnamurthy, A., Karp, R. M. \& Shamir, R. DEGAS: de novo discovery of dysregulated pathways in human diseases. PLOS ONE 5, e13367 (2010).

14. Manschwetus, J. T. et al. Binding of the human 14-3-3 isoforms to distinct sites in the leucine-rich repeat kinase 2. Front Neurosci. 14, 302 (2020).

15. Sato, S. et al. 14-3-3eta is a novel regulator of parkin ubiquitin ligase. EMBO J. 25, 211-221 (2006).

16. Yacoubian, T. A. et al. Differential neuroprotective effects of 14-3-3 proteins in models of Parkinson's disease. Cell Death Dis. 1, e2 (2010).

17. Plotegher, N. et al. The chaperone-like protein 14-3-3eta interacts with human alpha-synuclein aggregation intermediates rerouting the amyloidogenic pathway and reducing alpha-synuclein cellular toxicity. Hum. Mol. Genet. 23, 5615-5629 (2014).

18. van Heusden, G. P. et al. The 14-3-3 proteins encoded by the BMH1 and BMH2 genes are essential in the yeast Saccharomyces cerevisiae and can be replaced by a plant homologue. Eur. J. Biochem. 229, 45-53 (1995).

19. Aitken, A., Howell, S., Jones, D., Madrazo, J. \& Patel, Y. 14-3-3 alpha and delta are the phosphorylated forms of raf-activating 14-3-3 beta and zeta. In vivo stoichiometric phosphorylation in brain at a Ser-Pro-Glu-Lys MOTIF. J. Biol. Chem. 270, 5706-5709 (1995).

20. Berg, D., Holzmann, C. \& Riess, O. 14-3-3 proteins in the nervous system. Nat. Rev. Neurosci. 4, 752-762 (2003).

21. Wang, W. \& Shakes, D. C. Molecular evolution of the 14-3-3 protein family. J. Mol. Evol. 43, 384-398 (1996).

22. van Heusden, G. P., van der Zanden, A. L., Ferl, R. J. \& Steensma, H. Y. Four Arabidopsis thaliana 14-3-3 protein isoforms can complement the lethal yeast bmh1 bmh2 double disruption. FEBS Lett. 391, 252-256 (1996).

23. Fernandez, A., Tzeng, Y. H. \& Hsu, S. B. Subfunctionalization reduces the fitness cost of gene duplication in humans by buffering dosage imbalances. BMC Genomics 12, 604 (2011).

24. Uhart, M. \& Bustos, D. M. Human 14-3-3 paralogs differences uncovered by cross-talk of phosphorylation and lysine acetylation. PLoS One 8, e55703 (2013).

25. Shen, Y. H. et al. Significance of 14-3-3 self-dimerization for phosphorylationdependent target binding. Mol. Biol. Cell 14, 4721-4733 (2003).

26. Sluchanko, N. N. \& Gusev, N. B. Oligomeric structure of 14-3-3 protein: what do we know about monomers? FEBS Lett. 586, 4249-4256 (2012).

27. Yang, X. et al. Structural basis for protein-protein interactions in the 14-3-3 protein family. Proc. Natl Acad. Sci. USA 103, 17237-17242 (2006).

28. Han, D. et al. Functional identification of a novel 14-3-3 epsilon splicing variant suggests dimerization is not necessary for 14-3-3 epsilon to inhibit UV-induced apoptosis. Biochem Biophys. Res. Commun. 396, 401-406 (2010).

29. Liu, D. et al. Crystal structure of the zeta isoform of the 14-3-3 protein. Nature 376, 191-194 (1995).

30. Sluchanko, N. N. \& Uversky, V. N. Hidden disorder propensity of the N-terminal segment of universal adapter protein 14-3-3 is manifested in its monomeric form: Novel insights into protein dimerization and multifunctionality. Biochim Biophys. Acta 1854, 492-504 (2015).

31. Gardino, A. K., Smerdon, S. J. \& Yaffe, M. B. Structural determinants of 14-3-3 binding specificities and regulation of subcellular localization of 14-3-3-ligand complexes: a comparison of the X-ray crystal structures of all human 14-3-3 isoforms. Semin Cancer Biol. 16, 173-182 (2006).

32. Yaffe, M. B. et al. The structural basis for 14-3-3:phosphopeptide binding specificity. Cell 91, 961-971 (1997).

33. Rittinger, K. et al. Structural analysis of 14-3-3 phosphopeptide complexes identifies a dual role for the nuclear export signal of 14-3-3 in ligand binding. Mol. Cell 4, 153-166 (1999). 
34. Woodcock, J. M. et al. Role of salt bridges in the dimer interface of 14-3-3zeta in dimer dynamics, N-terminal alpha-helical order, and molecular chaperone activity. J. Biol. Chem. 293, 89-99 (2018).

35. Ferl, R. J., Manak, M. S. \& Reyes, M. F. The 14-3-3s. Genome Biol. 3, REVIEWS3010 (2002).

36. Sluchanko, N. N., Sudnitsyna, M. V., Seit-Nebi, A. S., Antson, A. A. \& Gusev, N. B. Properties of the monomeric form of human 14-3-3zeta protein and its interaction with tau and HspB6. Biochemistry 50, 9797-9808 (2011).

37. Wilker, E. W., Grant, R. A., Artim, S. C. \& Yaffe, M. B. A structural basis for 14-33sigma functional specificity. J. Biol. Chem. 280, 18891-18898 (2005).

38. Michaud, N. R., Fabian, J. R., Mathes, K. D. \& Morrison, D. K. 14-3-3 is not essential for Raf- 1 function: identification of Raf- 1 proteins that are biologically activated in a 14-3-3- and Ras-independent manner. Mol. Cell Biol. 15, 3390-3397 (1995).

39. Furukawa, Y. et al. Demonstration of the phosphorylation-dependent interaction of tryptophan hydroxylase with the 14-3-3 protein. Biochem. Biophys. Res Commun. 194, 144-149 (1993).

40. Ichimura, T. et al. Identification of the site of interaction of the 14-3-3 protein with phosphorylated tryptophan hydroxylase. J. Biol. Chem. 270, 28515-28518 (1995).

41. Yaffe, M. B. How do 14-3-3 proteins work?-Gatekeeper phosphorylation and the molecular anvil hypothesis. FEBS Lett. 513, 53-57 (2002).

42. Stevers, L. M., de Vink, P. J., Ottmann, C., Huskens, J. \& Brunsveld, L. A thermodynamic model for multivalency in 14-3-3 protein-protein interactions. J. Am Chem. Soc. 140, 14498-14510 (2018).

43. Obsil, T., Ghirlando, R., Klein, D. C., Ganguly, S. \& Dyda, F. Crystal structure of the 14-3-3zeta:serotonin $\mathrm{N}$-acetyltransferase complex. a role for scaffolding in enzyme regulation. Cell 105, 257-267 (2001).

44. Sekimoto, T., Fukumoto, M. \& Yoneda, Y. 14-3-3 suppresses the nuclear localization of threonine 157-phosphorylated p27(Kip1). EMBO J. 23, 1934-1942 (2004).

45. Fujita, N., Sato, S., Katayama, K. \& Tsuruo, T. Akt-dependent phosphorylation of p27Kip1 promotes binding to 14-3-3 and cytoplasmic localization. J. Biol. Chem. 277, 28706-28713 (2002)

46. Rezabkova, L. et al. 14-3-3 protein interacts with and affects the structure of RGS domain of regulator of G protein signaling 3 (RGS3). J. Struct. Biol. 170, 451-461 (2010).

47. Chiang, C. W. et al. Protein phosphatase $2 A$ activates the proapoptotic function of $B A D$ in interleukin- 3-dependent lymphoid cells by a mechanism requiring 14-3-3 dissociation. Blood 97, 1289-1297 (2001).

48. Corbit, K. C. et al. Activation of Raf-1 signaling by protein kinase $\mathrm{C}$ through a mechanism involving Raf kinase inhibitory protein. J. Biol. Chem. 278 13061-13068 (2003)

49. Van Der Hoeven, P. C., Van Der Wal, J. C., Ruurs, P., Van Dijk, M. C. \& Van Blitterswijk, J. 14-3-3 isotypes facilitate coupling of protein kinase C-zeta to Raf1: negative regulation by 14-3-3 phosphorylation. Biochem J. 345(Pt 2), 297-306 (2000).

50. Karlberg, T. et al. 14-3-3 proteins activate Pseudomonas exotoxins-S and -T by chaperoning a hydrophobic surface. Nat. Commun. 9, 3785 (2018).

51. Lin, J. P., Fan, Y. K. \& Liu, H. M. The 14-3-3eta chaperone protein promotes antiviral innate immunity via facilitating MDA5 oligomerization and intracellular redistribution. PLoS Pathog. 15, e1007582 (2019).

52. Wang, B. et al. 14-3-3 Proteins reduce cell-to-cell transfer and propagation of pathogenic alpha-synuclein. J. Neurosci. 38, 8211-8232 (2018).

53. $\mathrm{Xu}, \mathrm{Z}$. et al. 14-3-3 protein targets misfolded chaperone-associated proteins to aggresomes. J. Cell Sci. 126, 4173-4186 (2013).

54. Sluchanko, N. N. \& Gusev, N. B. Moonlighting chaperone-like activity of the universal regulatory 14-3-3 proteins. FEBS J. 284, 1279-1295 (2017).

55. Underwood, R. et al. 14-3-3 mitigates alpha-synuclein aggregation and toxicity in the in vivo preformed fibril model. Acta Neuropathol. Commun. 9, 13 (2021).

56. Ubl, A. et al. 14-3-3 protein is a component of Lewy bodies in Parkinson's disease-mutation analysis and association studies of 14-3-3 eta. Mol. Brain Res. 108, 33-39 (2002).

57. Williams, D. M. et al. NMR spectroscopy of 14-3-3zeta reveals a flexible C-terminal extension: differentiation of the chaperone and phosphoserinebinding activities of 14-3-3zeta. Biochem. J. 437, 493-503 (2011).

58. Sluchanko, N. N., Roman, S. G., Chebotareva, N. A. \& Gusev, N. B. Chaperone-like activity of monomeric human 14-3-3zeta on different protein substrates. Arch. Biochem. Biophys. 549, 32-39 (2014).

59. Liang, X. et al. Phosphorylation-dependent 14-3-3 protein interactions regulate CFTR biogenesis. Mol. Biol. Cell 23, 996-1009 (2012).

60. Yano, M., Nakamuta, S., Wu, X., Okumura, Y. \& Kido, H. A novel function of 14-3-3 protein: 14-3-3zeta is a heat-shock-related molecular chaperone that dissolves thermal-aggregated proteins. Mol. Biol. Cell 17, 4769-4779 (2006).

61. Aitken, A. Post-translational modification of 14-3-3 isoforms and regulation of cellular function. Semin Cell Dev. Biol. 22, 673-680 (2011).
62. Pennington, K. L., Chan, T. Y., Torres, M. P. \& Andersen, J. L. The dynamic and stressadaptive signaling hub of 14-3-3: emerging mechanisms of regulation and contextdependent protein-protein interactions. Oncogene 37, 5587-5604 (2018).

63. Civiero, L. et al. PAK6 Phosphorylates 14-3-3gamma to Regulate Steady State Phosphorylation of LRRK2. Front. Mol. Neurosci. 10, 417 (2017).

64. Woodcock, J. M. et al. Sphingosine and FTY720 directly bind pro-survival 14-3-3 proteins to regulate their function. Cell Signal 22, 1291-1299 (2010).

65. Dubois, T. et al. Structure and sites of phosphorylation of 14-3-3 protein: role in coordinating signal transduction pathways. J. Protein Chem. 16, 513-522 (1997).

66. Tsuruta, F. et al. JNK promotes Bax translocation to mitochondria through phosphorylation of 14-3-3 proteins. EMBO J. 23, 1889-1899 (2004).

67. Rommel, C. et al. Activated Ras displaces 14-3-3 protein from the amino terminus of c-Raf-1. Oncogene 12, 609-619 (1996).

68. Obsilova, V. et al. 14-3-3zeta C-terminal stretch changes its conformation upon ligand binding and phosphorylation at Thr232. J. Biol. Chem. 279, 4531-4540 (2004).

69. Choudhary, C. et al. Lysine acetylation targets protein complexes and coregulates major cellular functions. Science 325, 834-840 (2009).

70. Mortenson, J. B. et al. Histone deacetylase 6 (HDAC6) promotes the pro-survival activity of 14-3-3zeta via deacetylation of lysines within the 14-3-3zeta binding pocket. J. Biol. Chem. 290, 12487-12496 (2015).

71. Lodygin, D. \& Hermeking, H. The role of epigenetic inactivation of 14-3-3sigma in human cancer. Cell Res. 15, 237-246 (2005).

72. Ferguson, A. T. et al. High frequency of hypermethylation at the 14-3-3 sigma locus leads to gene silencing in breast cancer. Proc. Natl Acad. Sci. USA 97, 6049-6054 (2000).

73. Iwata, N. et al. Frequent hypermethylation of CpG islands and loss of expression of the 14-3-3 sigma gene in human hepatocellular carcinoma. Oncogene 19, 5298-5302 (2000)

74. Gasco, M. et al. Epigenetic inactivation of 14-3-3 sigma in oral carcinoma: association with p16(INK4a) silencing and human papillomavirus negativity. Cancer Res 62, 2072-2076 (2002).

75. Hermeking, H. The 14-3-3 cancer connection. Nat. Rev. Cancer 3, 931-943 (2003)

76. Margolis, S. S. et al. Role for the PP2A/B56delta phosphatase in regulating 14-3-3 release from Cdc25 to control mitosis. Cell 127, 759-773 (2006).

77. Margolis, S. S. et al. PP1 control of M phase entry exerted through 14-3-3regulated Cdc25 dephosphorylation. EMBO J. 22, 5734-5745 (2003).

78. Sluchanko, N. N. et al. Structural basis for the interaction of a human small heat shock protein with the 14-3-3 universal signaling regulator. Structure 25, 305-316 (2017).

79. Tzivion, G., Luo, Z. J. \& Avruch, J. Calyculin A-induced vimentin phosphorylation sequesters 14-3-3 and displaces other 14-3-3 partners in vivo. J. Biol. Chem. 275, 29772-29778 (2000)

80. Lee, D. H. et al. Role of glial 14-3-3 gamma protein in autoimmune demyelination. J. Neuroinflammation 12, 187 (2015).

81. Li, H. et al. 14-3-3gamma affects dynamics and integrity of glial filaments by binding to phosphorylated GFAP. J. Cell Sci. 119, 4452-4461 (2006).

82. Martin, H., Rostas, J., Patel, Y. \& Aitken, A. Subcellular localisation of 14-3-3 isoforms in rat brain using specific antibodies. J. Neurochem. 63, 2259-2265 (1994).

83. Cornell, B. \& Toyo-Oka, K. 14-3-3 proteins in brain development: neurogenesis, neuronal migration and neuromorphogenesis. Front. Mol. Neurosci. 10, 318 (2017).

84. Kaplan, A., Kent, C. B., Charron, F. \& Fournier, A. E. Switching responses: spatial and temporal regulators of axon guidance. Mol. Neurobiol. 49, 1077-1086 (2014).

85. Skoulakis, E. M. \& Davis, R. L. 14-3-3 proteins in neuronal development and function. Mol. Neurobiol. 16, 269-284 (1998).

86. Schroder, M. S. et al. Regulation of presynaptic anchoring of the scaffold protein Bassoon by phosphorylation-dependent interaction with 14-3-3 adaptor proteins. PLOS ONE 8, e58814 (2013).

87. Foote, M., Qiao, H., Graham, K., Wu, Y. \& Zhou, Y. Inhibition of 14-3-3 proteins leads to schizophrenia-related behavioral phenotypes and synaptic defects in mice. Biol. Psychiatry 78, 386-395 (2015).

88. Qiao, H., Foote, M., Graham, K., Wu, Y. \& Zhou, Y. 14-3-3 proteins are required for hippocampal long-term potentiation and associative learning and memory. $J$. Neurosci. 34, 4801-4808 (2014).

89. Wang, J., Lou, H., Pedersen, C. J., Smith, A. D. \& Perez, R. G. 14-3-3zeta contributes to tyrosine hydroxylase activity in MN9D cells: localization of dopamine regulatory proteins to mitochondria. J. Biol. Chem. 284, 14011-14019 (2009).

90. Hippenmeyer, S. et al. Genetic mosaic dissection of Lis1 and Ndel1 in neuronal migration. Neuron 68, 695-709 (2010).

91. Yingling, J., Toyo-Oka, K. \& Wynshaw-Boris, A. Miller-Dieker syndrome: analysis of a human contiguous gene syndrome in the mouse. Am. J. Hum. Genet 73 , 475-488 (2003). 
92. Toyo-oka, K. et al. 14-3-3epsilon is important for neuronal migration by binding to NUDEL: a molecular explanation for Miller-Dieker syndrome. Nat. Genet 34, 274-285 (2003).

93. Cheah, P. S. et al. Neurodevelopmental and neuropsychiatric behaviour defects arise from 14-3-3zeta deficiency. Mol. Psychiatry 17, 451-466 (2012).

94. Yoon, B. C., Zivraj, K. H., Strochlic, L. \& Holt, C. E. 14-3-3 Proteins regulate retinal axon growth by modulating ADF/cofilin activity. Dev. Neurobiol. 72, 600-614 (2012).

95. Kent, C. B. et al. 14-3-3 proteins regulate protein kinase a activity to modulate growth cone turning responses. J. Neurosci. 30, 14059-14067 (2010).

96. Kaplan, A. et al. Small-molecule stabilization of 14-3-3 protein-protein interactions stimulates axon regeneration. Neuron 93, 1082-1093 e1085 (2017).

97. Norkett, R., Del Castillo, U., Lu, W. \& Gelfand, V. I. Ser/Thr kinase Trc controls neurite outgrowth in Drosophila by modulating microtubule-microtubule sliding. Elife 9, https://doi.org/10.7554/eLife.52009 (2020).

98. Lavalley, N. J., Slone, S. R., Ding, H., West, A. B. \& Yacoubian, T. A. 14-3-3 Proteins regulate mutant LRRK2 kinase activity and neurite shortening. Hum. Mol. Genet. 25, 109-122 (2016)

99. Dhillon, A. S. et al. The C-terminus of Raf-1 acts as a 14-3-3-dependent activation switch. Cell Signal. 21, 1645-1651 (2009).

100. Kajiwara, Y., Buxbaum, J. D. \& Grice, D. E. SLITRK1 binds 14-3-3 and regulates neurite outgrowth in a phosphorylation-dependent manner. Biol. Psychiatry 66, 918-925 (2009).

101. Marzinke, M. A., Mavencamp, T., Duratinsky, J. \& Clagett-Dame, M. 14-3-3epsilon and NAV2 interact to regulate neurite outgrowth and axon elongation. Arch. Biochem. Biophys. 540, 94-100 (2013).

102. Ramser, E. M., Buck, F., Schachner, M. \& Tilling, T. Binding of alphall spectrin to 14-3-3beta is involved in NCAM-dependent neurite outgrowth. Mol. Cell Neurosci. 45, 66-74 (2010)

103. Ramser, E. M. et al. The 14-3-3zeta protein binds to the cell adhesion molecule L1, promotes L1 phosphorylation by CKII and influences L1-dependent neurite outgrowth. PLOS ONE 5, e13462 (2010).

104. Li, Y., Wu, Y. \& Zhou, Y. Modulation of inactivation properties of CaV2.2 channels by 14-3-3 proteins. Neuron $\mathbf{5 1}, 755-771$ (2006).

105. Piniella, D. et al. Identification of novel regulatory partners of the glutamate transporter GLT-1. Glia 66, 2737-2755 (2018).

106. Iovino, L., Tremblay, M. E. \& Civiero, L. Glutamate-induced excitotoxicity in Parkinson's disease: the role of glial cells. J. Pharm. Sci. 144, 151-164 (2020).

107. Zhang, J. \& Zhou, Y. 14-3-3 proteins in glutamatergic synapses. Neural Plast. 2018, 8407609 (2018).

108. Chen, B. S. \& Roche, K. W. Growth factor-dependent trafficking of cerebellar NMDA receptors via protein kinase B/Akt phosphorylation of NR2C. Neuron 62, 471-478 (2009).

109. Chung, C., Wu, W. H. \& Chen, B. S. Identification of novel 14-3-3 residues that are critical for isoform-specific interaction with GluN2C to regulate N-methyl-Daspartate (NMDA) receptor trafficking. J. Biol. Chem. 290, 23188-23200 (2015).

110. Ghorbani, S., Szigetvari, P. D., Haavik, J. \& Kleppe, R. Serine 19 phosphorylation and 14-3-3 binding regulate phosphorylation and dephosphorylation of tyrosine hydroxylase on serine 31 and serine 40. J. Neurochem. 152, 29-47 (2020).

111. Haavik, J. \& Toska, K. Tyrosine hydroxylase and Parkinson's disease. Mol. Neurobiol. 16, 285-309 (1998).

112. Tabrez, S. et al. A synopsis on the role of tyrosine hydroxylase in Parkinson's disease. CNS Neurol. Disord. Drug Targets 11, 395-409 (2012).

113. Johnson, M. E., Salvatore, M. F., Maiolo, S. A. \& Bobrovskaya, L. Tyrosine hydroxylase as a sentinel for central and peripheral tissue responses in Parkinson's progression: Evidence from clinical studies and neurotoxin models. Prog. Neurobiol. 165-167, 1-25 (2018).

114. Duty, S. \& Jenner, P. Animal models of Parkinson's disease: a source of novel treatments and clues to the cause of the disease. Br. J. Pharm. 164, 1357-1391 (2011).

115. van der Vlag, M., Havekes, R. \& Heckman, P. R. A. The contribution of Parkin, PINK1 and DJ-1 genes to selective neuronal degeneration in Parkinson's disease. Eur. J. Neurosci. 52, 3256-3268 (2020).

116. Soto-Ortolaza, A. I. et al. GWAS risk factors in Parkinson's disease: LRRK2 coding variation and genetic interaction with PARK16. Am. J. Neurodegener. Dis. 2, 287-299 (2013).

117. Dawson, T. M., Ko, H. S. \& Dawson, V. L. Genetic animal models of Parkinson's disease. Neuron 66, 646-661 (2010).

118. Gibb, W. R. \& Lees, A. J. The relevance of the Lewy body to the pathogenesis of idiopathic Parkinson's disease. J. Neurol. Neurosurg. Psychiatry 51, 745-752 (1988).

119. Shults, C. W. Lewy bodies. Proc. Natl Acad. Sci. USA 103, 1661-1668 (2006).

120. Spillantini, M. G., Crowther, R. A., Jakes, R., Hasegawa, M. \& Goedert, M. alphaSynuclein in filamentous inclusions of Lewy bodies from Parkinson's disease and dementia with lewy bodies. Proc. Natl Acad. Sci. USA 95, 6469-6473 (1998).
121. Shahmoradian, S. H. et al. Lewy pathology in Parkinson's disease consists of crowded organelles and lipid membranes. Nat. Neurosci. 22, 1099-1109 (2019).

122. Kuru, S., Yoshida, M., Tatsumi, S. \& Mimuro, M. Immunohistochemical localization of spatacsin in alpha-synucleinopathies. Neuropathology 34, 135-139 (2014).

123. Wakabayashi, K. et al. The Lewy body in Parkinson's disease and related neurodegenerative disorders. Mol. Neurobiol. 47, 495-508 (2013).

124. Kawamoto, Y. et al. 14-3-3 proteins in Lewy bodies in Parkinson disease and diffuse Lewy body disease brains. J. Neuropathol. Exp. Neurol. 61, 245-253 (2002).

125. Faber, I. et al. SPG11-related parkinsonism: clinical profile, molecular imaging and I-dopa response. Mov. Disord. 33, 1650-1656 (2018).

126. Cogo, S. et al. PKA-mediated phosphorylation of SPG11/spatacsin regulates binding with a subset of $14-3-3$ proteins. Preprint at bioRxiv https://doi.org/ 10.1101/2020.09.09.289009 (2020).

127. Ding, H., Fineberg, N. S., Gray, M. \& Yacoubian, T. A. alpha-Synuclein overexpression represses 14-3-3theta transcription. J. Mol. Neurosci. 51, 1000-1009 (2013).

128. Slone, S. R., Lavalley, N., McFerrin, M., Wang, B. \& Yacoubian, T. A. Increased 14-33 phosphorylation observed in Parkinson's disease reduces neuroprotective potential of 14-3-3 proteins. Neurobiol. Dis. 79, 1-13 (2015).

129. Slone, S. R., Lesort, M. \& Yacoubian, T. A. 14-3-3theta protects against neurotoxicity in a cellular Parkinson's disease model through inhibition of the apoptotic factor Bax. PLoS ONE 6, e21720 (2011).

130. McFerrin, M. B., Chi, X., Cutter, G. \& Yacoubian, T. A. Dysregulation of 14-3-3 proteins in neurodegenerative diseases with Lewy body or Alzheimer pathology. Ann. Clin. Transl. Neurol. 4, 466-477 (2017).

131. Terheyden, S., Ho, F. Y., Gilsbach, B. K., Wittinghofer, A. \& Kortholt, A. Revisiting the Roco G-protein cycle. Biochem. J. 465, 139-147 (2015).

132. Biosa, A. et al. GTPase activity regulates kinase activity and cellular phenotypes of Parkinson's disease-associated LRRK2. Hum. Mol. Genet. 22, 1140-1156 (2013).

133. Reynolds, A., Doggett, E. A., Riddle, S. M., Lebakken, C. S. \& Nichols, R. J. LRRK2 kinase activity and biology are not uniformly predicted by its autophosphorylation and cellular phosphorylation site status. Front. Mol. Neurosci. 7, 54 (2014).

134. Mills, R. D., Mulhern, T. D., Cheng, H. C. \& Culvenor, J. G. Analysis of LRRK2 accessory repeat domains: prediction of repeat length, number and sites of Parkinson's disease mutations. Biochem. Soc. Trans. 40, 1086-1089 (2012).

135. Monfrini, E. \& Di Fonzo, A. Leucine-rich repeat kinase (LRRK2) genetics and Parkinson's disease. Adv. Neurobiol. 14, 3-30 (2017).

136. Healy, D. G. et al. Phenotype, genotype, and worldwide genetic penetrance of LRRK2-associated Parkinson's disease: a case-control study. Lancet Neurol. 7, 583-590 (2008).

137. Navarrete, C., Martinez, I. \& Salamanca, F. Paternal line of transmission in chorea of Huntington with very early onset. Genet. Couns. 5, 175-178 (1994).

138. West, A. B. et al. Parkinson's disease-associated mutations in leucine-rich repeat kinase 2 augment kinase activity. Proc. Natl Acad. Sci. USA 102, 16842-16847 (2005).

139. Gilsbach, B. K. et al. Roco kinase structures give insights into the mechanism of Parkinson disease-related leucine-rich-repeat kinase 2 mutations. Proc. Natl Acad. Sci. USA 109, 10322-10327 (2012).

140. lannotta, L. et al. Divergent effects of G2019S and R1441C LRRK2 mutations on LRRK2 and Rab10 phosphorylations in mouse tissues. Cells 9, https://doi.org/ 10.3390/cells9112344 (2020).

141. Kania, E. \& Parys, J. B. The emerging interrelation between ROCO and related kinases, intracellular $\mathrm{Ca}(2+)$ signaling, and autophagy. Biochim. Biophys. Acta Mol. Cell Res. 1866, 1054-1067 (2019).

142. Lewis, P. A. et al. The R1441C mutation of LRRK2 disrupts GTP hydrolysis. Biochem. Biophys. Res. Commun. 357, 668-671 (2007).

143. Liao, J. et al. Parkinson disease-associated mutation R1441H in LRRK2 prolongs the "active state" of its GTPase domain. Proc. Natl Acad. Sci. USA 111, 4055-4060 (2014).

144. Sheng, Z. et al. Ser1292 autophosphorylation is an indicator of LRRK2 kinase activity and contributes to the cellular effects of PD mutations. Sci. Transl. Med. 4, 164ra161 (2012)

145. Kluss, J. H. et al. Detection of endogenous S1292 LRRK2 autophosphorylation in mouse tissue as a readout for kinase activity. NPJ Parkinsons Dis. 4, 13 (2018).

146. Wang, S. et al. Elevated LRRK2 autophosphorylation in brain-derived and peripheral exosomes in LRRK2 mutation carriers. Acta Neuropathol. Commun. 5, 86 (2017).

147. Nichols, R. J. et al. 14-3-3 binding to LRRK2 is disrupted by multiple Parkinson's disease-associated mutations and regulates cytoplasmic localization. Biochem. J. 430, 393-404 (2010).

148. Kostelecky, B., Saurin, A. T., Purkiss, A., Parker, P. J. \& McDonald, N. Q. Recognition of an intra-chain tandem 14-3-3 binding site within PKCepsilon. EMBO Rep. 10, 983-989 (2009).

149. Molzan, M. \& Ottmann, C. Synergistic binding of the phosphorylated S233- and S259-binding sites of C-RAF to one 14-3-3zeta dimer. J. Mol. Biol. 423, 486-495 (2012). 
150. Stevers, L. M. et al. Structural interface between LRRK2 and 14-3-3 protein. Biochem. J. 474, 1273-1287 (2017).

151. Stevers, L. M. et al. Characterization and small-molecule stabilization of the multisite tandem binding between 14-3-3 and the R domain of CFTR. Proc. Nat Acad. Sci. USA 113, E1152-E1161 (2016).

152. Sluchanko, N. N. Reading the phosphorylation code: binding of the 14-3-3 protein to multivalent client phosphoproteins. Biochem. J. 477, 1219-1225 (2020).

153. Dzamko, N. et al. Inhibition of LRRK2 kinase activity leads to dephosphorylation of $\operatorname{Ser}(910) / \operatorname{Ser}(935)$, disruption of $14-3-3$ binding and altered cytoplasmic localization. Biochem. J. 430, 405-413 (2010).

154. Li, X. et al. Phosphorylation-dependent 14-3-3 binding to LRRK2 is impaired by common mutations of familial Parkinson's disease. PLOS ONE 6, e17153 (2011).

155. Dzamko, N. et al. The IkappaB kinase family phosphorylates the Parkinson's disease kinase LRRK2 at Ser935 and Ser910 during Toll-like receptor signaling. PLOS ONE 7, e39132 (2012).

156. Chia, R. et al. Phosphorylation of LRRK2 by casein kinase 1alpha regulates transGolgi clustering via differential interaction with ARHGEF7. Nat. Commun. 5, 5827 (2014).

157. Lobbestael, E. et al. Pharmacological LRRK2 kinase inhibition induces LRRK2 protein destabilization and proteasomal degradation. Sci. Rep. 6, 33897 (2016).

158. Muda, K. et al. Parkinson-related LRRK2 mutation R1441C/G/H impairs PKA phosphorylation of LRRK2 and disrupts its interaction with 14-3-3. Proc. Nat/ Acad. Sci. USA 111, E34-E43 (2014).

159. Fraser, K. B. et al. LRRK2 secretion in exosomes is regulated by 14-3-3. Hum. Mol. Genet. 22, 4988-5000 (2013).

160. Di Maio, R. et al. LRRK2 activation in idiopathic Parkinson's disease. Sci. Transl. Med. 10, eaar5429 (2018).

161. Zhao, J., Molitor, T. P., Langston, J. W. \& Nichols, R. J. LRRK2 dephosphorylation increases its ubiquitination. Biochem. J. 469, 107-120 (2015).

162. Clayton, D. F. \& George, J. M. The synucleins: a family of proteins involved in synaptic function, plasticity, neurodegeneration and disease. Trends Neurosci. 21, 249-254 (1998).

163. Appel-Cresswell, S. et al. Alpha-synuclein p.H50Q, a novel pathogenic mutation for Parkinson's disease. Mov. Disord. 28, 811-813 (2013).

164. Chartier-Harlin, M. C. et al. Alpha-synuclein locus duplication as a cause of familial Parkinson's disease. Lancet 364, 1167-1169 (2004).

165. Kruger, R. et al. Ala30Pro mutation in the gene encoding alpha-synuclein in Parkinson's disease. Nat. Genet. 18, 106-108 (1998).

166. Lesage, S. et al. G51D alpha-synuclein mutation causes a novel parkinsonianpyramidal syndrome. Ann. Neurol. 73, 459-471 (2013).

167. Polymeropoulos, M. H. et al. Mutation in the alpha-synuclein gene identified in families with Parkinson's disease. Science 276, 2045-2047 (1997).

168. Singleton, A. B. et al. alpha-Synuclein locus triplication causes Parkinson's disease. Science 302, 841 (2003).

169. Zarranz, J. J. et al. The new mutation, E46K, of alpha-synuclein causes Parkinson and Lewy body dementia. Ann. Neurol. 55, 164-173 (2004).

170. Souza, J. M., Giasson, B. I., Chen, Q., Lee, V. M. \& Ischiropoulos, H. Dityrosine cross-linking promotes formation of stable alpha -synuclein polymers. Implication of nitrative and oxidative stress in the pathogenesis of neurodegenerative synucleinopathies. J. Biol. Chem. 275, 18344-18349 (2000).

171. Auluck, P. K., Chan, H. Y., Trojanowski, J. Q., Lee, V. M. \& Bonini, N. M. Chaperone suppression of alpha-synuclein toxicity in a Drosophila model for Parkinson's disease. Science 295, 865-868 (2002)

172. Feany, M. B. \& Bender, W. W. A Drosophila model of Parkinson's disease. Nature 404, 394-398 (2000).

173. Thakur, P. et al. Modeling Parkinson's disease pathology by combination of fibril seeds and alpha-synuclein overexpression in the rat brain. Proc. Natl Acad. Sci. USA 114, E8284-E8293 (2017).

174. Ostrerova, N. et al. alpha-Synuclein shares physical and functional homology with 14-3-3 proteins. J. Neurosci. 19, 5782-5791 (1999).

175. Muntane, G., Ferrer, I. \& Martinez-Vicente, M. alpha-synuclein phosphorylation and truncation are normal events in the adult human brain. Neuroscience 200, 106-119 (2012).

176. Okochi, M. et al. Constitutive phosphorylation of the Parkinson's disease associated alpha-synuclein. J. Biol. Chem. 275, 390-397 (2000).

177. Ellis, C. E., Schwartzberg, P. L., Grider, T. L., Fink, D. W. \& Nussbaum, R. L. alphasynuclein is phosphorylated by members of the Src family of protein-tyrosine kinases. J. Biol. Chem. 276, 3879-3884 (2001).

178. Fujiwara, $\mathrm{H}$. et al. alpha-Synuclein is phosphorylated in synucleinopathy lesions. Nat. Cell Biol. 4, 160-164 (2002).

179. Takahashi, M. et al. Phosphorylation of alpha-synuclein characteristic of synucleinopathy lesions is recapitulated in alpha-synuclein transgenic Drosophila. Neurosci. Lett. 336, 155-158 (2003).
180. Oueslati, A. Implication of alpha-synuclein phosphorylation at S129 in synucleinopathies: what have we learned in the last decade? J. Parkinsons Dis. 6 , 39-51 (2016).

181. Alam, M. \& Schmidt, W. J. Rotenone destroys dopaminergic neurons and induces parkinsonian symptoms in rats. Behav. Brain Res. 136, 317-324 (2002).

182. Sherer, T. B. et al. Mechanism of toxicity in rotenone models of Parkinson's disease. J. Neurosci. 23, 10756-10764 (2003).

183. Abounit, S., Wu, J. W., Duff, K., Victoria, G. S. \& Zurzolo, C. Tunneling nanotubes: a possible highway in the spreading of tau and other prion-like proteins in neurodegenerative diseases. Prion 10, 344-351 (2016).

184. Li, J. Y. et al. Lewy bodies in grafted neurons in subjects with Parkinson's disease suggest host-to-graft disease propagation. Nat. Med. 14, 501-503 (2008).

185. Olanow, C. W. \& Brundin, P. Parkinson's disease and alpha synuclein: is Parkinson's disease a prion-like disorder? Mov. Disord. 28, 31-40 (2013)

186. Masuda-Suzukake, M. et al. Prion-like spreading of pathological alpha-synuclein in brain. Brain 136, 1128-1138 (2013).

187. Henderson, M. X., Trojanowski, J. Q. \& Lee, V. M. alpha-Synuclein pathology in Parkinson's disease and related alpha-synucleinopathies. Neurosci. Lett. 709, 134316 (2019).

188. Braak, H. \& Braak, E. Neuropathological stageing of Alzheimer-related changes. Acta Neuropathol. 82, 239-259 (1991).

189. $\mathrm{Xu}$, J. et al. Dopamine-dependent neurotoxicity of alpha-synuclein: a mechanism for selective neurodegeneration in Parkinson disease. Nat. Med. 8, 600-606 (2002).

190. Sarraf, S. A. et al. Landscape of the PARKIN-dependent ubiquitylome in response to mitochondrial depolarization. Nature 496, 372-376 (2013).

191. Seirafi, M., Kozlov, G. \& Gehring, K. Parkin structure and function. FEBS J. 282, 2076-2088 (2015).

192. Pilcher, H. Parkin implicated in sporadic Parkinson's disease. Lancet Neurol. 4, 798 (2005).

193. Kitada, T. et al. Mutations in the parkin gene cause autosomal recessive juvenile parkinsonism. Nature 392, 605-608 (1998).

194. Johansen, K. K., Torp, S. H., Farrer, M. J., Gustavsson, E. K. \& Aasly, J. O. A case of Parkinson's disease with no lewy body pathology due to a homozygous exon deletion in parkin. Case Rep. Neurol. Med. 2018, 6838965 (2018).

195. Jesko, H., Lenkiewicz, A. M., Wilkaniec, A. \& Adamczyk, A. The interplay between parkin and alpha-synuclein; possible implications for the pathogenesis of Parkinson's disease. Acta Neurobiol. Exp. 79, 276-289 (2019).

196. Shimura, H. et al. Ubiquitination of a new form of alpha-synuclein by parkin from human brain: implications for Parkinson's disease. Science 293, 263-269 (2001).

197. Martina, J. A., Chen, Y., Gucek, M. \& Puertollano, R. MTORC1 functions as a transcriptional regulator of autophagy by preventing nuclear transport of TFEB. Autophagy 8, 903-914 (2012).

198. Nezich, C. L., Wang, C., Fogel, A. I. \& Youle, R. J. MiT/TFE transcription factors are activated during mitophagy downstream of Parkin and Atg5. J. Cell Biol. 210, 435-450 (2015).

199. Yaffe, M. B. et al. A motif-based profile scanning approach for genome-wide prediction of signaling pathways. Nat. Biotechnol. 19, 348-353 (2001).

200. Obenauer, J. C., Cantley, L. C. \& Yaffe, M. B. Scansite 2.0: Proteome-wide prediction of cell signaling interactions using short sequence motifs. Nucleic Acids Res 31, 3635-3641 (2003).

201. Puntervoll, $P$. et al. ELM server: a new resource for investigating short functional sites in modular eukaryotic proteins. Nucleic Acids Res. 31, 3625-3630 (2003).

202. Miller, M. L. et al. Linear motif atlas for phosphorylation-dependent signaling. Sci. Signal 1, ra2 (2008).

203. Tinti, M. et al. ANIA: ANnotation and Integrated Analysis of the 14-3-3 interactome. Database 2014, bat085 (2014)

204. Madeira, F. et al. 14-3-3-Pred: improved methods to predict 14-3-3-binding phosphopeptides. Bioinformatics 31, 2276-2283 (2015).

205. Ottmann, C. et al. A structural rationale for selective stabilization of antitumor interactions of 14-3-3 proteins by cotylenin A. J. Mol. Biol. 386, 913-919 (2009).

206. Sijbesma, E. et al. Identification of two secondary ligand binding sites in 14-3-3 proteins using fragment screening. Biochemistry 56, 3972-3982 (2017).

207. Andrei, S. A. et al. Stabilization of protein-protein interactions in drug discovery. Expert Opin. Drug Disco. 12, 925-940 (2017).

208. Stevers, L. M. et al. Modulators of 14-3-3 protein-protein interactions. J. Med. Chem. 61, 3755-3778 (2018).

209. Thiel, P., Kaiser, M. \& Ottmann, C. Small-molecule stabilization of protein-protein interactions: an underestimated concept in drug discovery? Angew. Chem. Int Ed. Engl. 51, 2012-2018 (2012).

210. Wang, B. et al. Isolation of high-affinity peptide antagonists of 14-3-3 proteins by phage display. Biochemistry 38, 12499-12504 (1999). 
211. Dong, S. et al. 14-3-3 Integrates prosurvival signals mediated by the AKT and MAPK pathways in ZNF198-FGFR1-transformed hematopoietic cells. Blood 110, 360-369 (2007).

212. Masters, S. C. \& Fu, H. 14-3-3 proteins mediate an essential anti-apoptotic signal. J. Biol. Chem. 276, 45193-45200 (2001).

213. Yan, Y. et al. Implication of 14-3-3epsilon and 14-3-3theta/tau in proteasome inhibition-induced apoptosis of glioma cells. Cancer Sci. 104, 55-61 (2013).

214. Kaplan, A., Ottmann, C. \& Fournier, A. E. 14-3-3 adaptor protein-protein interactions as therapeutic targets for CNS diseases. Pharm. Res. 125, 114-121 (2017).

215. Takahashi, T. et al. Synergistic combination therapy with cotylenin A and vincristine in multiple myeloma models. Int. J. Oncol. 46, 1801-1809 (2015).

216. Molzan, M. et al. Stabilization of physical RAF/14-3-3 interaction by cotylenin A as treatment strategy for RAS mutant cancers. ACS Chem. Biol. 8, 1869-1875 (2013).

217. Anders, C. et al. A semisynthetic fusicoccane stabilizes a protein-protein interaction and enhances the expression of $\mathrm{K}+$ channels at the cell surface. Chem. Biol. 20, 583-593 (2013).

218. Bier, D. et al. Small-molecule stabilization of the $14-3-3 /$ Gab2 protein-protein interaction (PPI) interface. ChemMedChem 11, 911-918 (2016).

219. de Vink, P. J. et al. Cooperativity basis for small-molecule stabilization of proteinprotein interactions. Chem. Sci. 10, 2869-2874 (2019).

220. Rose, R. et al. Identification and structure of small-molecule stabilizers of 14-3-3 protein-protein interactions. Angew. Chem. Int. Ed. Engl. 49, 4129-4132 (2010).

221. Kaplan, A. et al. Polypharmacological perturbation of the 14-3-3 adaptor protein interactome stimulates neurite outgrowth. Cell Chem. Biol. 27, 657-667 e656 (2020).

222. Mabonga, L. \& Kappo, A. P. Protein-protein interaction modulators: advances, successes and remaining challenges. Biophys. Rev. 11, 559-581 (2019).

223. Kathman, S. G. \& Statsyuk, A. V. Covalent tethering of fragments for covalent probe discovery. Medchemcomm 7, 576-585 (2016).

224. Hartman, A. M. et al. Discovery of small-molecule stabilizers of 14-3-3 proteinprotein interactions via dynamic combinatorial chemistry. ACS Med. Chem. Lett. 11, 1041-1046 (2020).

225. Pair, F. S. \& Yacoubian, T. A. 14-3-3 proteins: novel pharmacological targets in neurodegenerative diseases. Trends Pharm. Sci. https://doi.org/10.1016/j. tips.2021.01.001 (2021).

226. Kast, D. J. \& Dominguez, R. Mechanism of IRSp53 inhibition by 14-3-3. Nat. Commun. 10, 483 (2019).

\section{ACKNOWLEDGEMENTS}

University of Padova to support L.C. as assistant professor and the IRCCS San Camillo Hospital in Venice, Italy. This work was supported by UniPD (STARs 2019: Supporting
TAlents in ReSearch), the Italian Ministry of Health (GR-2016-02363461) to L.C., and the IRCCS San Camillo Hospital in Venice, Italy. T.A.Y. was supported by the NIH/ NINDS grants R01NS112203, R56NS115767, and P50NS108675. We are also grateful to the Michael J Fox Foundation for supporting E.G.

\section{AUTHOR CONTRIBUTIONS}

L.C. and E.Gi. conceived and wrote the manuscript. T.A.Y. and E.Gr. contributed to writing and revising the manuscript. All authors read and approved the final manuscript.

\section{COMPETING INTERESTS}

The authors declare no competing interests.

\section{ADDITIONAL INFORMATION}

Correspondence and requests for materials should be addressed to L. Civiero.

Reprints and permission information is available at http://www.nature.com/ reprints

Publisher's note Springer Nature remains neutral with regard to jurisdictional claims in published maps and institutional affiliations.

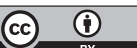

Open Access This article is licensed under a Creative Commons Attribution 4.0 International License, which permits use, sharing, adaptation, distribution and reproduction in any medium or format, as long as you give appropriate credit to the original author(s) and the source, provide a link to the Creative Commons license, and indicate if changes were made. The images or other third party material in this article are included in the article's Creative Commons license, unless indicated otherwise in a credit line to the material. If material is not included in the article's Creative Commons license and your intended use is not permitted by statutory regulation or exceeds the permitted use, you will need to obtain permission directly from the copyright holder. To view a copy of this license, visit http://creativecommons. org/licenses/by/4.0/.

(c) The Author(s) 2021 